\title{
Quadrature Mirror Filter Banks, M-Band Extensions and Perfect-Reconstruction Techniques
}

\section{P. P. Vaidyanathan}

\section{Abstract}

In this paper, quadrature mirror filters (QMF) are reviewed. After a brief introduction to multirate building blocks, the two-band QMF bank is discussed. Various distortions caused by the structure, and methods to eliminate these distortions are outlined. Perfect-reconstruction structures for the twoband case are reviewed, and the results are extended to the case of arbitrary number of channels. The relation between perfect-reconstruction QMF banks, and the concept of losslessness in transfer-matrices is indicated. New lattice structures are presented, which perform the perfect reconstruction, sometimes even under coefficient quantization.

\section{INTRODUCTION}

Q UADRATURE MIRROR FILJER (QMF) banks have been of great interest during the past decade, ever since their introduction by Croisier, Esteban and Galand [1], [2]. These find applications in situations where a discrete-time signal $x(n)$ is to be split into a number of consecutive bands in the frequency domain, so that each subband signal $x_{k}(n)$ can be processed in an independent manner. Typical 'processing' includes undersampling the subband signals, encoding them and transmitting over a channel, or merely storing the coded signals. Eventually, at some point in the process, the subband signals should somehow be recombined so that the original signal is properly reconstructed. Typical applications of such signal-splitting include subband coders for speech signals [2], [3], [29], digital transmultiplexers [26] used in FDM/TDM conversion, and frequency domain speechscramblers [30].

In this paper we look at the QMF problem purely as a signal-processing problem. The exact signal characteristics and the nature of the application are not given any emphasis. This blissful freedom from specific aspects of the external world enables us to concentrate on the basic science of the problem from the viewpoint of signalreconstruction. Our purpose here is to outline the signal- processing issues and mathematics involved. We address issues such as this: what kinds of errors are involved in the implementation of a QMF bank? Which of these errors can be reduced to acceptable levels? Which of these errors can be completely eliminated (at finite cost)? Is it possible to completely eliminate all errors that result due to the transmission of a signal through a QMF analysis/synthesis bank? If so, what constraints does this impose on the filters that take part in the filter-bank?

The QMF problem, basically simple looking, is in fact a fundamental signal reconstruction problem, and opens up several intriguing possibilities when addressed from a theoretical viewpoint. It is related, surprisingly, to the the ory of lattice structures and orthogonal matrix-functions, even though these relations are not explicit from the problem statement. Our attempt here is to tie up several apparently unrelated results in a unified manner, so as to place in evidence the enormous scope and implicit beauty of this problem.

\section{THE TWO CHANNEL QMF BANK}

The QMF bank is a multirate digital filter bank. The term multirate signifies that the sampling rate is not constant throughout the system [3]; there are decimators in the system which down-sample a sequence, and there are interpolators which perform up-sampling. Since decimators and interpolators are the building blocks of any multirate digital system, let us briefly review their characteristics. Details and proofs can be found in the text by Crochiere and Rabiner [3].

\section{Decimators}

An $M$-fold decimator is shown in Fig. 1(a). Its input is a sequence $x(n)$, and the output sequence $y(n)$ is a compressed version of $x(n)$. More specifically, the output is obtained by retaining only those samples of $x(n)$ which occur at times which are multiples of the integer $M$. The input-output relation is $y(n)=x(M n)$. The figure demonstrates the decimation operation for $M=2$ and also shows that a decimator is a time-varying device, even though it is 
linear. Accordingly, it cannot be represented by a transfer function; we should seek other means of describing the decimator in the transform-domain. Since a decimator causes a compression in the time-domain, we expect a 'stretching' in the frequency domain. For example, with $M=2$, the quantity $X\left(\mathrm{e}^{j \omega / 2}\right)$ is a stretched version of $X\left(\mathrm{e}^{j \omega}\right)$. However, since $X\left(e^{j \omega / 2}\right)$ has a period of $4 \pi$ rather than $2 \pi$, it is not a valid transform of a sequence. It can be verified that $Y\left(e^{j \omega}\right)$ in fact has two terms. The first is $X\left(e^{j \omega / 2}\right)$, and the second term is $X\left(-e^{j \omega / 2}\right)$ which is a shifted version of the first term (shifted by an amount $2 \pi$ ). More formally, the input-output relation for a two-fold decimator can be written in the transform domain as [3]

$$
Y\left(e^{j \omega}\right)=0.5\left[X\left(e^{j \omega / 2}\right)+X\left(-e^{j \omega / 2}\right)\right] .
$$

Note that $Y\left(e^{j \omega}\right)$ given as above does have a period of $2 \pi$. This is demonstrated in Fig. 1(b), (c) where $x(n)$ is assumed to be a lowpass type of signal. If the transform of $x(n)$ is

(a)

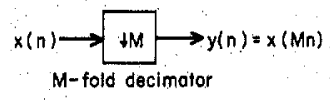

\begin{tabular}{|c|c|c|c|c|c|c|c|c|c|}
\hline \multicolumn{10}{|c|}{ Example $(M=2)$} \\
\hline $\begin{array}{l}n \\
x(n) \\
y(n)\end{array}$ & $\begin{array}{l}0 \\
1 \\
1\end{array}$ & $\begin{array}{r}1 \\
.8 \\
8\end{array}$ & $\begin{array}{l}2 \\
8 \\
2\end{array}$ & $\begin{array}{r}3 \\
-1 \\
3\end{array}$ & $\begin{array}{l}4 \\
2 \\
?\end{array}$ & $\begin{array}{r}5 \\
6 \\
.\end{array}$ & $\begin{array}{l}6 \\
3\end{array}$ & $\begin{array}{r}7 \\
15\end{array}$ & $\ldots$ \\
\hline $\begin{array}{l}x^{\prime}(n) \\
y^{\prime}(n)\end{array}$ & $\begin{array}{l}0 \\
0\end{array}$ & $\begin{array}{l}1 \\
4\end{array}$ & $\begin{array}{c}4 \\
-1\end{array}$ & $\begin{array}{l}8 \\
6\end{array}$ & & 2 & 6 & & $\begin{array}{l}\text { shlfted input) } \\
\text { new out put) }\end{array}$ \\
\hline
\end{tabular}

(b)

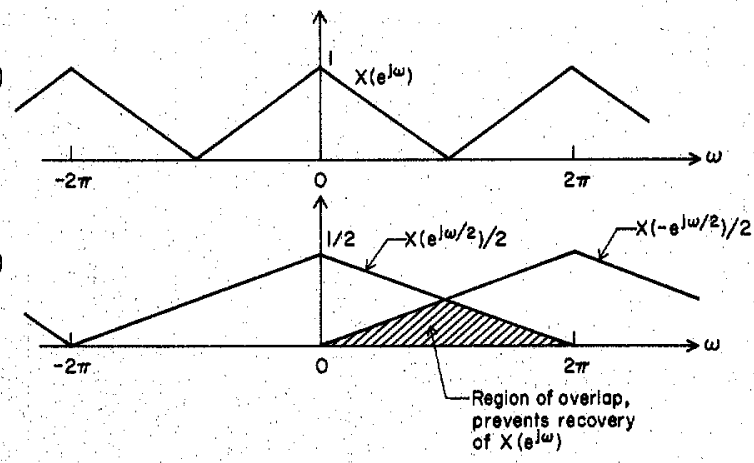

Figure 1. A decimator is a system which takes in a sequence $x(n)$ and produces a time-compressed sequence $y[n]=x(M n)$. The compression ratio $M$ is an integer. This operation is denoted by the downgoing arrow lindicative of down-sampling]. The figure demonstrates the operation of a two-fold decimator. In general the output $y(n)$ of an $M$-fold decimator is obtained by retaining only the samples of the input sequence occurring at times that are integermultiples of $M$. A decimator is a time-varying system (even though linear). The figure demonstrates this, by showing that a shifted version of the input does not produce a shifted version of the output. The effect of compression in the time domain is an expansion cor stretching in the frequency domain. The quantity $\frac{1}{2} X\left(e^{j \omega / 2}\right)$ in the figure represents this, while $\frac{1}{2} X\left(-e^{j \omega / 2}\right)$ represents aliasing caused by downsampling. For arbitrary $M$, equation [2] gives the transform of the decimated sequence. not bandlimited to $-\pi / 2 \leq \omega \leq \pi / 2$, there is an overlap of the two terms in (1) as shown by the shaded area in Fig. 1(c). This overlap is the aliasing effect, caused by undersampling. There is ro way we can get back the original signal $x(n)$ from $y(n)$, once aliasing has taken place. In order to convince ourself that the scale factor of 0.5 is required in the expression (1), let us imagine, as an example, that $X(n)$ is the unit pulse $\delta(n)$. Then $X(z)$ is unity for all values of the argument $z$, hence from (1) we have $Y\left(e^{j \omega}\right)=0.5(1+1)=1.0$ for all $\omega$, which is consistent with the fact that $y(n)=\delta(n)$ in this case.

For an $M$-fold decimator we have $y(n)=x(M n)$, and the transform-domain relation is precisely an extension of (1). Instead of two terms, we now have $M$ terms; the first term is merely a stretched version of $X\left(e^{j \omega}\right)$ (by a factor of $M)$, namely $X\left(e^{j \omega / M}\right)$. The remaining terms are uniformly shifted versions of the first term (the amount of shift being integer multiples of $2 \pi$ ). Thus, it can be shown that

$Y\left(\mathrm{e}^{j \omega}\right)=\frac{1}{M} \sum_{k=0}^{M-1} X\left(\mathrm{e}^{j(\omega-2 \pi k) / M}\right), \quad Y(z)=\frac{1}{M} \sum_{k=0}^{M-1} X\left(z^{1 / M} W^{k}\right)$

where $W=e^{-2 \pi / M}$ and $z=e^{j \omega}$. The scale factor of $1 / M$ in (2) can be understood in a manner analogous to the factor 0.5 in (1).

What happens if we decimate a highpass signal, say by

(a)

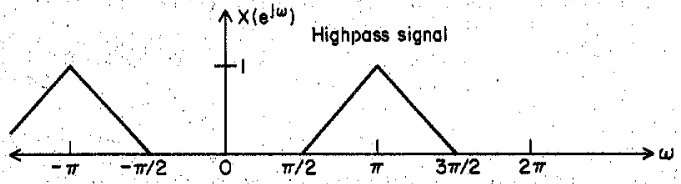

(b)

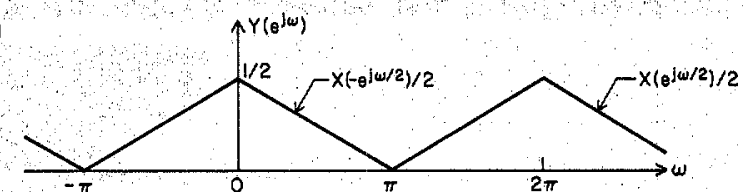

(c)

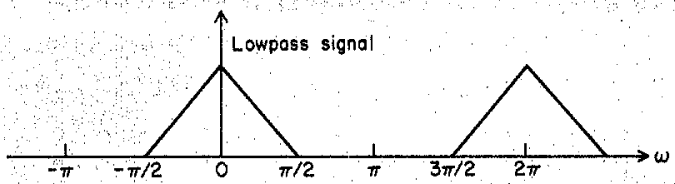

Figure 2. What happens when a highpass signal gets decimated? Fig. $2(a)$ represents the transform $X\left(e^{j \omega}\right)$ (assumed to be real for simplicity) of a highpass signal. If this signal is decimated two-fold, the resulting transform has two components, proportional to $X\left(e^{j \omega / 2}\right)$ and $X\left(-e^{i w / 2}\right)$, as shown in Fig. $2(b)$. Accordingly, the decimated signal looks like a regular lowpass signal. In fact if a lowpass signal with transform as in Fig. $2(c)$ were decimated by a factor of two, the result would again have appearance as in Fig. $2[\mathrm{~b}]$. This means that given the transform in Fig. $2[b]$, it is impossible to tell whether is came from Fig. $2(a)$ or $2(c)$. 
a factor of two? Since it is not bandlimited to $-\pi / 2 \leq \omega \leq$ $\pi / 2$, the first impression is that there will be aliasing. Let us refer to Fig. 2, Assuming the highpass signal to be bandlimited to $\pi / 2 \leq \omega \leq 3 \pi / 2$ (Fig. 2(a)), the decimator output $Y\left(e^{j \omega}\right)$ is as shown in Fig. 2(b). Thus, the decimated version of the highpass signal looks like a lowpass signal, Notice that if a signal having a lowpass spectrum as in Fig. 2(c) were decimated, we would obtain precisely the spectrum of Fig. 2(b) again. In other words, simply by looking at Fig. 2(b), it is not possible to tell whether it came from Fig. 2(a) or Fig. 2(c). This is a kind of aliasing; theoretically speaking, we have undersampled a highpass signal. However, since there is no overlap between the two curves in Fig. 2(b), we can, in principle, reconstruct the signal $x(n)$ from $y(n)$, as long as we have the additional information as to whether $x(n)$ is a lowpass or highpass signal.

In practice, before a signal is passed through a decimator, it is first bandlimited (by using a bandpass filter, of which lowpass filtering is a special case), so as to reduce the effects of aliasing. Such filters are called decimation filters.

\section{Interpolators}

An $M$-fold interpolator, schematically shown in Fig. 3, inserts $M-1$ zeros between adjacent samples. Its input output relation is given in Fig. 3 . The effect of this stretching in the time domain is a compression in the frequency domain, as demonstrated in Fig. $3(b)$. Since $Y\left(e^{j \omega}\right)$ has $M-1$ replica (or images) of the basic prototype spectrum, the interpolator is said to cause an imaging effect (which is the dual of the aliasing effect of a decimator). The transform domain relation is simple, given by

$$
Y\left(e^{j \omega}\right)=X\left(e^{j \omega M}\right), \quad Y(z)=X\left(z^{M}\right) .
$$

It can be verified through simple examples, that an interpolator is also a time-varying, linear system.

In practice, an interpolator is followed by a filter called the interpolation filter, which eliminates the images in Fig. 3 so that the result is a simple bandpass signal for lowpass signal, as desired).

It is interesting to see what happens when a decimator and an interpolator are cascaded (Fig. 4(a)). The decimator causes stretching and aliasing, whereas the interpolator causes compression, all in the frequency domain. The end result is shown in Fig. 4 (c). If the spectrum of $x_{k}(n)$ is bandlimited to $-\pi / M \leq \omega \leq \pi / M$, then Fig. 4 (c) is an imaged version of Fig. 4(b) with no stretching or compression.

Notice in passing, that if the decimator and interpolator in the cascade are interchanged, the result is just an identity system; the decimator simply gets rid of the zeros thrown in by the interpolator.

\section{The Two-Channel Quadrature Mirror Filter (QMF) Bank}

The two-channel QMF bank, shown in Fig. 5, is one of the earliest and most commonly employed structures [1], [2]. The analysis bank is composed of a lowpass filter $H_{0}(z)$ and a highpass filter $H_{1}(z)$, which split the incoming sequence $x(n)$ into two frequency bands. The lowpass signal $x_{0}(n)$ and the highpass signal $x_{1}(n)$ are then decimated by factors of two. The decimated signals are typically encoded [31], and transmitted. At the receiver end, the signals are decoded, and passed through the interpolators. The decimator-interpolator cascade causes aliasing and imaging as discussed in Fig. 4 earlier. The purpose of the synthesis filters $F_{0}(z)$ and $F_{1}(z)$ is to eliminate the images. $F_{0}(z)$ is a lowpass filter so that the highpass image of the interpolated lowpass signal $x_{0}(n)$ is suppressed. Similarly, $F_{1}(z)$ is highpass so that the lowpass image of the interpolated highpass signal is eliminated. As a result, the signals $v_{0}(n)$ and $v_{1}(n)$ are good approximations of $x_{0}(n)$ and $x_{1}(n)$, and the reconstructed signal $\hat{X}(z)$ (hopefully) resembles $X(z)$ closely. Note in passing that the name quadrature mirror filter derives from the fact that, the response of $H_{1}(z)$ is the mirror-image of the response of $H_{0}(z)$, with respect to frequency $\pi / 2$ (which is a quarter of the sampling frequency).

Now what makes the QMF problem nontrivial and fascinating? In order to avoid aliasing (due to decimation), the responsés of $H_{0}(z)$ and $H_{i}(z)$ must be disjoint as in Fig. 6(a). On the other hand, in order that no frequency range shall be 'left out' by the analysis bank, the responses should be overlapping as in Fig. 6(b). The only obvious solution to this dilemma is to make the responses very sharp (approximating the ideal response in Fig. 6(c)), but

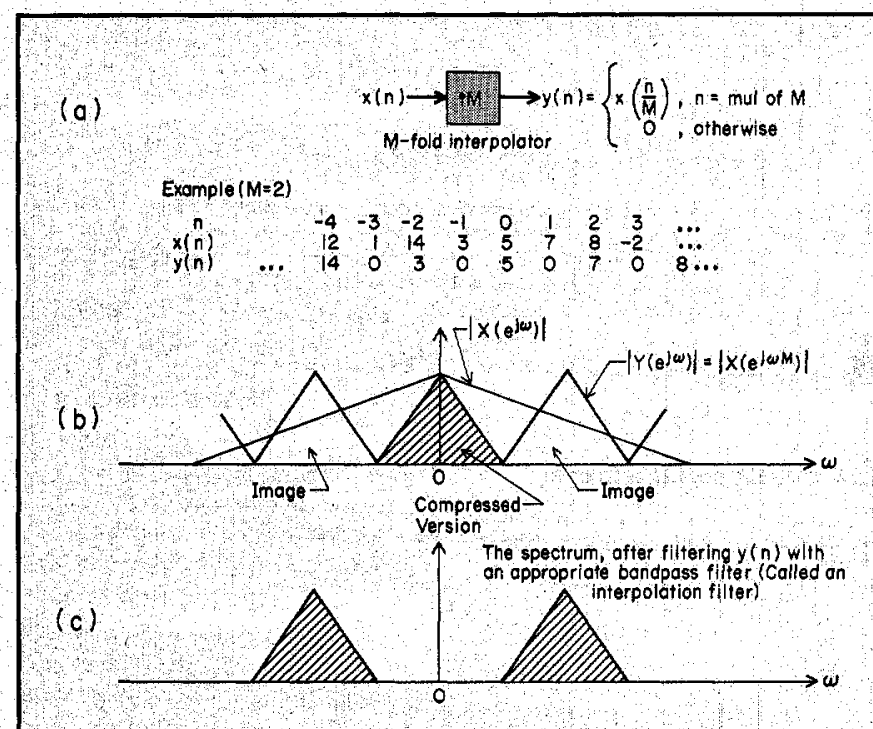

Figure 3. An $M$-fold interpolator merely inserts $M-1$ zero-valued samples between adjacent samples of the input sequence $x(n)$. An upgoing arrow with $M$ written on the side indicates this (Fig. $3(\mathrm{a})$ ). The effect of this stretching in the time domain is a compression in the frequency domain as shown. Thus, $M-1$ images of the original spectrum are caused by this operation. The term imaging is often used [3] to describe this, and is the dual of the aliasing effect caused by a decimator. Interpolators are linear, time-varying operators. An interpolation filter is an ordinary digital filter, which rejects the images in Fig. $3(b)$ and retains a single, desired image. For example, a bandpass filter can be used on the interpolated signal, to produce the spectrum of Fig. $3(\mathrm{c})$. 
it is well known that sharp cutoff filters require very high order, are highly sensitive to quantization, and often cause instability problems (if IIR).

The philosophy adopted [1], [2], [5] in the QMF bank in order to overcome this problem is to permit aliasing at the output of the decimator, by designing the analysis filters as in Fig. $6(b)$, and then choosing the synthesis filters $F_{0}(z)$ and $F_{1}(z)$ such that the imaging produced by the interpolators cancels the aliasing. In fact exact cancelation is possible. This observation relieves the designer of a very stringent analysis-filter design problem.

Based on the relations (2) and (3) for a decimator and an interpolator respectively, it is possible to express $\hat{X}(z)$ in Fig. 5 as

$$
\begin{aligned}
\hat{X}(z)= & \frac{1}{2}\left[H_{0}(z) F_{0}(z)+H_{1}(z) F_{1}(z)\right] X(z) \\
& +\frac{1}{2}\left[H_{0}(-z) F_{0}(z)+H_{1}(-z) F_{1}(z)\right] X(-z)
\end{aligned}
$$

Because of the second term in (4) involving $X(-z)$, we cannot write down an expression for $\hat{X}(z) / X(z)$ that is independent of $X(z)$ itself. This is not surprising, since the QMF bank is not time-invariant (as the decimators and interpolators are time-variant). The second term in (4) represents the effects of aliasing and imaging. This term can be made to disappear simply by choosing the synthesis filters to be

$$
F_{0}(z)=H_{1}(-z), \quad F_{1}(z)=-H_{0}(-z)
$$

Once the aliasing is so canceled, the QMF bank becomes a (linear and) time-invariant system with transfer function

$$
T(z)=\frac{\hat{X}(z)}{X(z)}=\frac{1}{2}\left[H_{0}(z) H_{1}(-z)-H_{1}(z) H_{0}(-z)\right]
$$

Ideally, we would like $T(z)$ to be a delay, i.e., $T(z)=z^{-n_{0}}$, so that the reconstructed signal is a delayed version of $x(n)$. Since $T(z)$ is in general not a delay, it represents a distortion and is called the distortion function or the overall transfer function. The quantity $\left|T\left(e^{j \omega}\right)\right|$ is the amplitude distortion and $\arg \left[T\left(\mathrm{e}^{j \omega}\right)\right]$ is the phase distortion. If $T(z)$ is an allpass function, i.e., if $\left|T\left(\mathrm{e}^{j \omega}\right)\right|=$ constant for all $\omega$, then there is no amplitude distortion. If $T(z)$ is a linearphase FIR function, then $\arg \left[T\left(\mathrm{e}^{j \omega}\right)\right]=K \omega$, and there is no phase distortion. Barnwell [29] has shown that there are several interesting ways in which the filters $H_{0}(z), H_{7}(z)$, $F_{0}(z)$ and $F_{1}(z)$ can be related, so as to obtain an appropriate functional form for $T(z)$.

It is typical to choose $H_{1}(z)=H_{0}(-z)$, so that we have a lowpass/highpass pair. Then

$$
T(z)=\frac{1}{2}\left[H_{0}^{2}(z)-H_{7}^{2}(z)\right]=\frac{1}{2}\left[H_{0}^{2}(z)-H_{0}^{2}(-z)\right]
$$

which represents the distortion function. Suppose $H_{0}(z)$ and $H_{1}(z)$ are linear phase FIR filters, then $T(z)$ given by (7) clearly has linear phase, and phase distortion is easily eliminated. In summary, the choice of transfer functions according to

$$
H_{1}(z)=H_{0}(-z), \quad F_{0}(z)=H_{0}(z), \quad F_{1}(z)=-H_{1}(z)
$$

leads to complete elimination of aliasing; if $H_{0}(z)$ has linear phase, then phase distortion is also eliminated. Assuming $H_{0}(z)$ to be a linear phase lowpass. FIR filter of order $N-1$, we can write $H_{0}\left(e^{j \omega}\right)=e^{-j \omega(N-1) / 2} H_{0, a}\left(e^{j \omega}\right)$ where $H_{0, a}\left(e^{j \omega}\right)$ is the (real-valued) amplitude response [32]. With this, $T\left(e^{j \omega}\right)$ takes on a nice form:

$$
T\left(\mathrm{e}^{j \omega}\right)=\frac{\mathrm{e}^{-j \omega(N-1)}}{2}\left[\left|H_{0}\left(\mathrm{e}^{j \omega}\right)\right|^{2}-(-1)^{N-1}\left|H_{1}\left(\mathrm{e}^{j \omega}\right)\right|^{2}\right]
$$

If $N-1$ is even, then referring to Fig. $6(\mathrm{~b})$, at the frequency $\omega=\pi / 2, T\left(\mathrm{e}^{j \omega}\right)$ given by (9) is zero! This implies severe amplitude distortion. Accordingly, with the choice of filters as in (8), we must always pick the order $N-1$ of the linear phase FIR filter $H_{0}(z)$ to be odd ${ }^{\ddagger}$. Equation (9) then yields

$$
\left|T\left(\mathrm{e}^{j \omega}\right)\right|=\frac{1}{2}\left[\left|H_{0}\left(\mathrm{e}^{j \omega}\right)\right|^{2}+\left|H_{1}\left(\mathrm{e}^{j \omega}\right)\right|^{2}\right]
$$

which represents the residual amplitude distortion. Since $T(z)$ has linear phase, phase-distortion is absent.

Now comes the bad news: if two linear phase transfer functions $H_{0}(z)$ and $H_{1}(z)$ are such that $\left|H_{0}\left(e^{j \omega}\right)\right|^{2}+$ $\left|H_{1}\left(\mathrm{e}^{j \omega}\right)\right|^{2}$ is constant for all $\omega$, then $H_{0}(z)$ and $H_{1}(z)$ must be trivial transfer functions [24] with frequency responses of the form $\left|H_{0}\left(e^{j \omega}\right)\right|^{2}=\cos ^{2}(K \omega)$ and $\left|H_{1}\left(e^{j \omega}\right)\right|^{2}=\sin ^{2}(K \omega)$. In

FIf an even order is required for some other reasons, there is a trick which can be employed to avoid the above distortion; see [5].

(a)

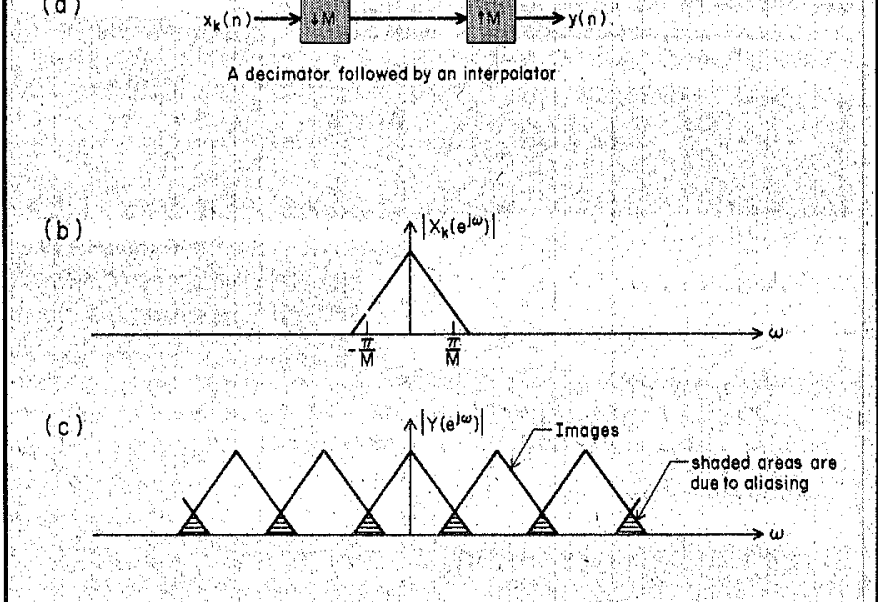

Figure 4. When a decimator is followed by an interpolator, aliasing and imaging effects are simultaneously. present; the stretching and aliasing caused by the decimator are followed up by the imaging process caused by the interpolator. It is not possible to recover $x_{k}(n)$ from $y(n)$, unless the shaded areas in Fig, $4(\mathrm{c})$ are absent. 
other words, for the choice of filters as in (8), there does not exist a non-trivial linear-phase transfer function $H_{0}(z)$ such that phase distortion and amplitude distortion are simultaneously eliminated!

To complement the above discussion, let us now see how amplitude distortion can be completely eliminated, when one agrees to tolerate phase distortion. There are many ways of doing this [11], [27], [29], [43]; let us look at one. In the world of IIR digital filters, there exists a beautiful subset of transfer functions which can be implemented as a sum of two allpass functions [33], [34], [39], [40], [43]. If such transfer functions are used in the analysis bank, this automatically forces $T(z)$ to be allpass. Without getting into the theoretical details, let us state the essence compactly here.

Let $H_{0}(z)$ be a lowpass IIR function with numerator polynomial $P(z)$ and denominator polynomial $D(z)$ of orders $N$, i.e.,

$$
H_{0}(z)=P(z) / D(z)=\sum_{n=0}^{N} p_{n} z^{-n} / \sum_{n=0}^{N} d_{n} z^{-n}
$$

A typical magnitude response of $H_{0}(z)$ is indicated in Fig. 8(a). Let us define the highpass function $H_{1}(z)$ to be such that $\left|H_{0}\left(e^{j \omega}\right)\right|^{2}+\left|H_{1}\left(e^{j \omega}\right)\right|^{2}=1$, for all $\omega$. Such a pair $\left[H_{0}(z), H_{1}(z)\right]$ is called a power-complementary pair. Given $H_{0}(z)$ such that $\left|H_{0}\left(e^{j \omega}\right)\right| \leq 1$, we can always find such $H_{1}(z)$ by defining it to be $H_{i}(z)=Q(z) / D(z)$ where $Q(z)$ is a spectral factor of

$$
\left|Q\left(\mathrm{e}^{j \omega}\right)\right|^{2}=\left|D\left(\mathrm{e}^{j \omega}\right)\right|^{2}-\left|P\left(\mathrm{e}^{j \omega}\right)\right|^{2}
$$

Usually, the zeros of $H_{0}(z)$ are on the unit circle, hence $P(z)$ is a symmetric polynomial, i.e., $p_{n}=p_{N-n}$. Moreover, it is

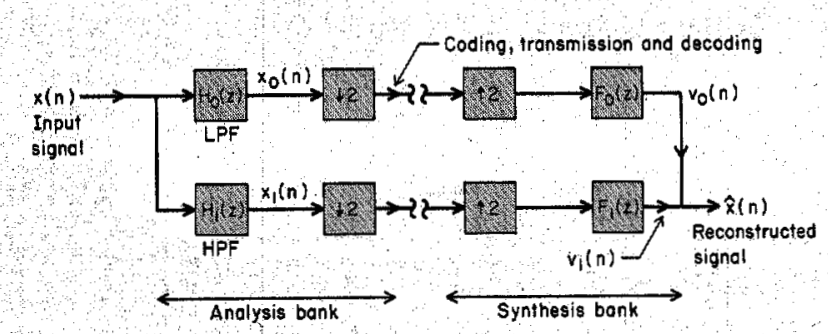

Figure 5. The two-channel Quadrature Mirror Filter (OMF) bank. Here, $H_{0}(z)$ and $H_{1}(z)$ represent lowpass and highpass filters respectively (called the analysis filters). The filtered signals $x_{0}(n)$ and $x_{1}(n)$ are decimated by a factor of two and transmitted, with possible encoding. At the receiver, the signals are interpolated lafter decoding if necessary, and filtered by $F_{0}(z)$ and $F_{1}(z)$ (called the synthesis filters), before recombination. The purpose of $H_{0}(z)$ and $H_{1}(z)$ is to make the two frequency-bands as independent as possible, while the purpose of $F_{0}(z)$ and $F_{1}(z)$ is to eliminate the images caused by the decimatorinterpolator system. The reconstructed signal $\hat{x}(n)$ is related to $x(n)$ by equation (4), where the term involving $X[-z]$ represents the effects of aliasing and imaging. The first term in this equation is a filtered version of $X(z)$. Ideally, we would like the second term to be zero, and the first term to be of the form $c z^{-n} \circ X(z)$, so that $x(n)$ is a (delayed) replica of $x(n)$.

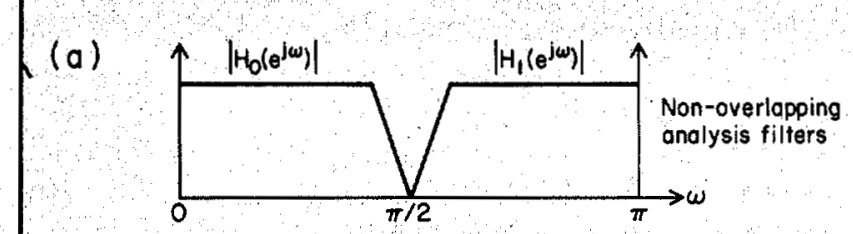

(b)

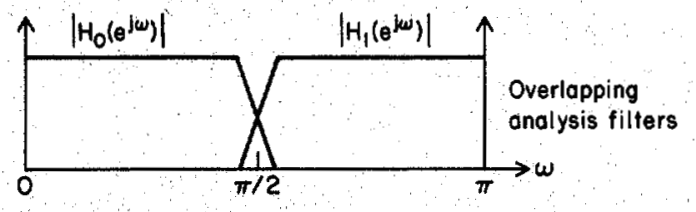

(c)

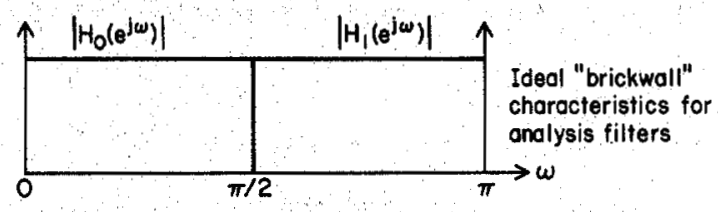

Figure 6 . In the two-channel QMF structure, it is a nontrivial task to design $H_{0}(z)$ and $H_{1}(z)$. If the responses are disjoint, as in Fig. G(a), then there is practically a spectral hole' which is undesirable. If they overlap as in Fig. 6(b), then there is aliasing due to subsequent decimation, because the filters have bandwidths exceeding $\pi / 2$. Since a brickwall response as in Fig. $G(c)$ is not practicable, the response shown in Fig. 6(b) is usually chosen. The aliasing caused by decimation is then canceled by judicious choice of the synthesis filters $F_{0}(z)$ and $F_{1}(z)$. If these filters are chosen as in equation (5) then aliasing is completely canceled (no matter how $H_{0}(z)$ and $H_{1}(z)$ are chosen) and the QMF system becomes a (linear and) time-invariant system, characterized by the transfer function $T(z)$ in $(6)$. $T(z)$ is called the distortion transfer function or the overall transfer function.

very common for $H_{1}(z)$ to have all zeros on the unit circle too (Fig. 8(a)). For example, if $H_{0}(z)$ is a digital Butterworth, Chebyshev or elliptic filter, this is always the case; accordingly, $Q(z)$ is a symmetric or antisymmetric polynomial. It is antisymmetric (i.e., $q_{n}=-q_{N-n}$ ) if $Q(z)$ has a zero at $\omega=0$, which usually is the case when $N$ is odd. Now, there is a result [34], [39] which says that if two transfer functions $H_{0}(z)=P(z) / D(z)$ and $H_{1}(z)=Q(z) / D(z)$ with symmetric $P(z)$ and antisymmetric $Q(z)$ are such that $\left|H_{0}\left(e^{j \omega}\right)\right|^{2}+\left|H_{1}\left(e^{j \omega}\right)\right|^{2}=1$, for all $\omega$, then they must be of the form

$$
H_{0}(z)=\left(A_{0}(z)+A_{1}(z)\right) / 2, \quad H_{1}(z)=\left(A_{0}(z)-A_{1}(z)\right) / 2
$$

where $A_{0}(z)$ and $A_{1}(z)$ are allpass functions. In other words, we can implement the analysis bank as in Fig. $8(b)$. Assume that in addition to all the above conditions; $H_{1}(z)$ also satisfies $H_{1}(z)=H_{0}(-z)$. You can ensure this by designing $H_{0}(z)$ such that the response $\left|H_{0}\left(e^{j \omega}\right)\right|^{2}$ has a symmetry around $\omega=\pi / 2$, i.e., $\omega_{p}+\omega_{s}=\pi$ and $\varepsilon_{1}=\varepsilon_{2}$ as shown in Fig. 8(c). Simply by employing such $H_{0}(z)$ and $H_{1}(z)$ in the QMF bank of Fig. 5, and picking $F_{0}(z)$ and $F_{1}(z)$ as in (5) so as to cancel aliasing, we end up with the 

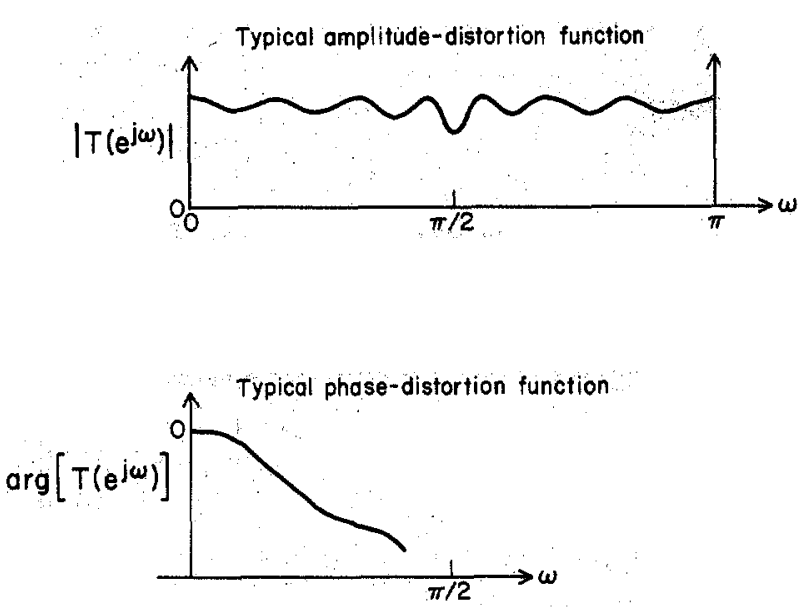

Figure 7. Once aliasing-error has been eliminated by choice of synthesis filters as in equation (5), the QMF bank suffers from two errors, viz., the amplitude distortion $\left|T\left(e^{j \omega}\right)\right|$ and the phase distortion $\arg \left|T\left(e^{j \omega}\right)\right|$, which can be evaluated from equation (7) when the filters are related by equation ( 8 ). There is no amplitude distortion if $\left.T\left(e^{j \omega}\right)\right]$ is constant for all $\omega$; there is no phase distortion if arg $\left|T\left(e^{j \omega}\right)\right|$ is of the form $K \omega$ for constant $K$. If $H_{0}(z)$ is a linear phase FIR filter, then $T(z)$ in (7) has linear-phase too. Phase distortion can therefore be completely eliminated. The remaining amplitude distortion is given by (10) with $(N-1)$ odd see text.

distortion function $T(z)=A_{0}(z) A_{1}(z) / 2$ which is allpass! In other words, amplitude distortion is completely eliminated (and so is aliasing, of course). The phase response of $T(z)$ leads to some phase distortion. A discussion on how to design transfer functions $H_{0}(z)$ and $H_{1}(z)$ satisfying $(13)$ and the condition $H_{1}(z)=H_{0}(-z)$, can be found in [11], [43].

It is worth pointing out some of the good features of implementations based on allpass decompositions, such as (13). The allpass filters $A_{0}(z)$ and $A_{1}(z)$ can be implemented using the Gray-and-Markel lattice structures [38] which come in various convenient forms. All of these forms can be made free from limit cycles [44] (which are parasitic oscillations under zero-input, caused by quantizer-nonlinearities in feedback loops of IIR filters). Moreover, instability problems due to coefficient quantization are absent in these lattice structures. It is also known that implementations based on the decomposition of (13) have low passband sensitivity [34] (even though this is not very crucial for QMF applications).

The relations (13) imply that $A_{0}(z)=H_{0}(z)+H_{1}(z)$, and $A_{1}(z)=H_{0}(z)-H_{1}(z)$. Since in addition $H_{1}(\dot{z})=H_{0}(-z)$, you can verify that $A_{0}(z)$ in fact has the form $a_{0}\left(z^{2}\right)$ and $A_{1}(z)$ has the form $z^{-1} a_{1}\left(z^{2}\right)$. Accordingly, the QMF bank can be implemented as in Fig. $8(d)$. If $a_{0}(z)$ and $a_{1}(z)$ are implemented using a cascade of the one-multiplier Gray-Markel lattice structure [38], then the entire QMF bank (i.e., all four transfer functions $H_{0}(z), H_{1}(z) ; F_{0}(z)$ and $\left.F_{1}(z)\right)$ can be implemented with a total of about $N$ multipliers, where $N$ is the order of $H_{0}(z)$ ! In other words, the structure of Fig. $8(d)$ is dramatically efficient.

\section{The Perfect-Reconstruction Two Channel QMF Bank}

So we see that either phase distortion or amplitude distortion can be completely eliminated, according to choice. The remaining distortion can be either minimized using computer-aided techniques, or equalized by cascading with a filter.

For example, once phase distortion has been eliminated, amplitude distortion can be minimized by use of nonlinear optimization software [23]. Usually, an objective function is formulated which is a sum of the stopband error of $H_{0}(z)$ and the amplitude distortion error $\int\left[\left|T\left(e^{j \omega}\right)\right|^{2}-1\right]^{2} d \omega$; the coefficients of $H_{0}(z)$ are found such that this objective function is minimized. The remaining filters $H_{1}(z), F_{0}(z)$ and $F_{1}(z)$ are found from (8). An

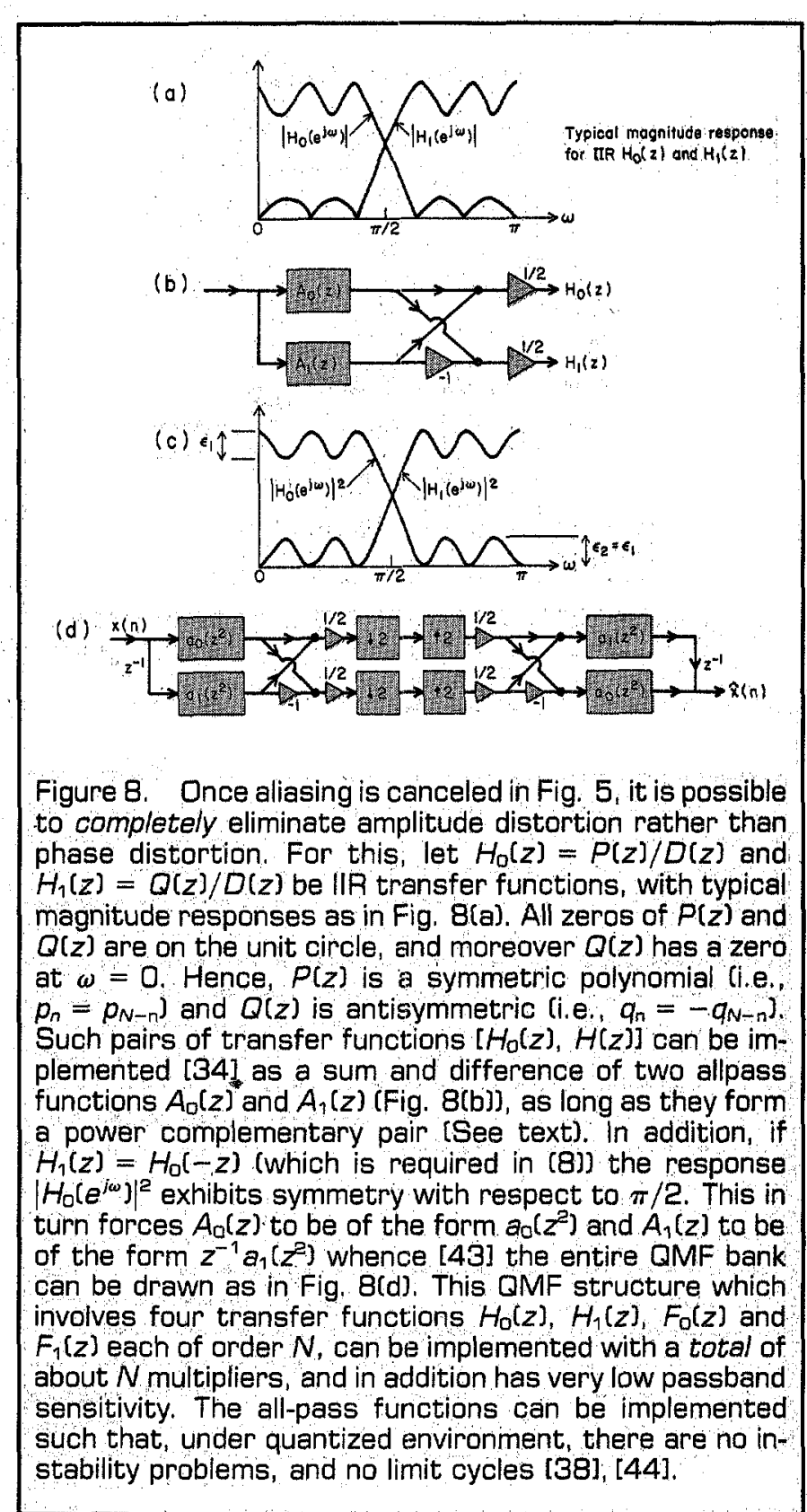


alternative to this optimization would be to cascade a linear-phase FIR filter to the output $\hat{x}(n)$ and equalize the amplitude distortion.

Now comes the crucial question: can we simultaneously eliminate both amplitude and phase distortions in a twochannel QMF bank? In an interesting article, smith and Barnwell have shown [6] that the answer is yes. Such a QMF bank is said to have perfect-reconstruction property, because $\hat{x}(n)$ is a replica of $x(n)$ except for a delay. We can accomplish this by exploiting a property of linear-phase FIR halfband filters. Assuming that the required order of $H_{0}(z)$ is $N-1$, let us first design a linear-phase FIR halfband filter $G(z)$ of order $2(N-1)$ with amplitude response $G_{a}\left(e^{j \omega}\right)$ as in Fig. $9(a)$; the response exhibits a symmetry with respect to $\pi / 2$ (i.e., the passband ripple is equal to the stop band ripple, and $\left.\omega_{p}+\omega_{S}=\pi\right)$. From $G(z)$ we can construct a new halfband filter $G_{+}(z)=G(z)+\delta z^{-(N-1)}$ so that the amplitude response of $C_{+}(z)$ is nonnegative (as shown in Fig. 9(b)). (The term 'amplitude response' has been defined earlier; see, for example, discussion preceding equation (9)). We can therefore find a spectral factor ${ }^{+} H_{0}(z)$ of $G_{+}(z)$ having only real coefficients. Because of symmetry around $\pi / 2$ we have,

$$
G_{+}(z)+(-1)^{N-1} G_{+}(-z)=d z^{-(N-1)}
$$

Fi.e, a function $H_{0}(z)$ such that $G_{+}(z)=z^{-(N-1)} H_{0}\left(z^{-1}\right) H_{0}(z)$.

(a)

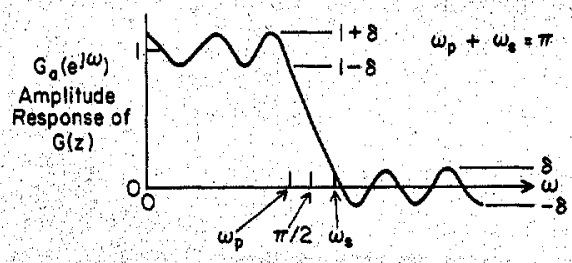

(b)

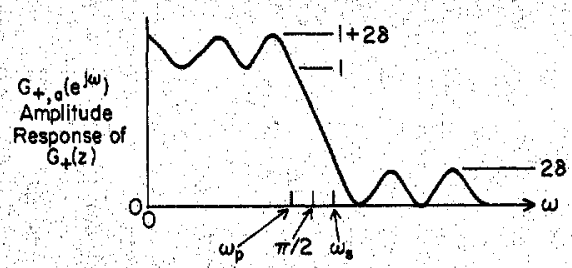

(c)

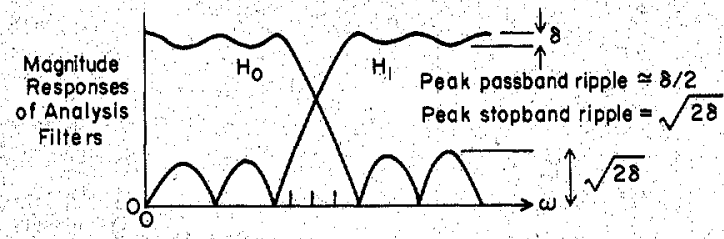

Figure 9. If the QMF bank is such that all three distortions (viz, aliasing, amplitude distortion and phase distortion) are eliminated, then we have $\hat{x}(n)=c x\left(n-n_{0}\right)$. Such a system is said to have Perfect Reconstruction. Property (PRP for short). For the two channel QMF bank, a system with PRP can be constructed by designing $H_{0}(z)$ to be a spectral factor of an appropriately conditioned FIR half-band filter, and then choosing the remaining transfer functions as in (16); see text. where $d$ is a constant, hence

$$
H_{0}\left(z^{-1}\right) H_{0}(z)+H_{0}\left(-z^{-1}\right) H_{0}(-z)=d .
$$

Our objective is to pick $H_{0}(z)$ and $H_{1}(z)$ such that, after aliasing has been canceled (by the choice of (5)), the distortion $T(z)$ in $(6)$ is a delay. Since $H_{0}(z)$ satisfies (15), we can accomplish our goal simply by choosing $N-1$ to be odd and $H_{1}(z)=z^{-(N-1)} H_{0}\left(-z^{-1}\right)$. This is therefore a neat solution to the perfect reconstruction problem! In summary, let $H_{0}(z)$ be a spectral factor of a linear-phase FIR half band filter $G_{+}(z)$ having a positive amplitude response. Choose the remaining filters according to

$$
\begin{gathered}
H_{1}(z)=z^{-(N-1)} H_{0}\left(-z^{-1}\right), \quad F_{0}(z)=z^{-(N-1)} H_{0}\left(z^{-1}\right) \\
F_{1}(z)=z^{-(N-1)} H_{1}\left(z^{-1}\right)
\end{gathered}
$$

Then we have perfect reconstruction in the QMF bank of Fig. 5 , and $\hat{x}(n)=c x(n-N+1)$, where $c$ is a constant. The expressions for $F_{0}(z)$ and $F_{i}(z)$ in (16) come from the alias-cancelation condition (5). It is important to notice that the stopband attenuation and stopband edge $\omega_{s}$ of $H_{0}(z)$ can be adjusted to any desired value, simply by

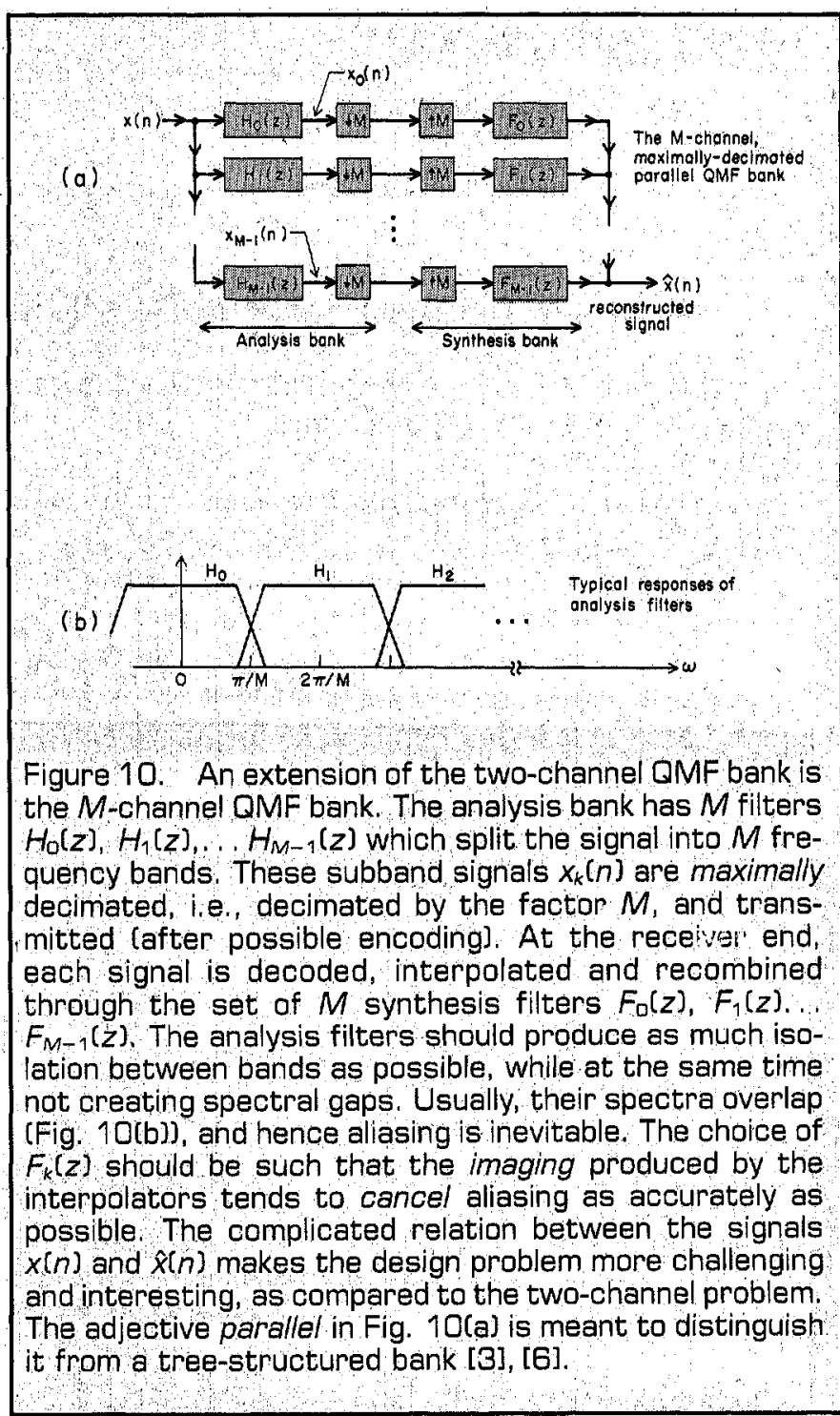


designing the halfband filter $G(z)$ appropriately, by using the McClellan-Parks algorithm [35].

\section{THE M-CHANNEL QMF BANK}

Figure 10 shows the $M$-channel QMF bank for arbitrary $M$. The analysis filters $H_{0}(z), H_{1}(z), \ldots H_{M-1}(z)$ split the signal $x(n)$ into $M$ frequency bands, and each subband signal $x_{k}(n)$ is decimated by a factor of $M$. This is called a maximally decimated structure because, the decimation factors are equal to $M$, which is the number of bands. The signals $x_{k}(n)$ are encoded and transmitted. At the synthesizer end, they are decoded, interpolated, and filtered by the synthesis filters $F_{0}(z), F_{1}(z), \ldots, F_{M-1}(z)$. The signal $\hat{x}(n)$ is a reconstructed version of $x(n)$, and we wish it to be 'close' to $x(n)$ in some sense.

In analogy with the two-channel QMF problem, $\hat{x}(n)$ suffers from three types of errors. First, there is aliasing. Figure 10(b) shows the typical analysis filter responses ${ }^{*}$. Since there is an overlap between adjacent filters, the signals are not strictly bandlimited to a sufficient extent. This causes aliasing. And then there are amplitude and phase distortions.

In absence of the decimators and interpolators, the relation between $\hat{X}(z)$ and $X(z)$ is nice and simple: $\hat{X}(z)=X(z) \sum_{k=0}^{M-1} H_{k}(z) F_{k}(z)$. The decimators and interpolators cause aliasing and imaging; by applying the relations in (2) and (3) we can arrive at the following key expression for $\hat{X}(z)$ :

$$
\hat{X}(z)=\frac{1}{M} \sum_{n=0}^{M-1} X\left(z W^{n}\right) \sum_{k=0}^{M-1} H_{k}\left(z W^{n}\right) F_{k}(z)
$$

The cutoff frequency of the lowpass filter $H_{0}(z)$ is not a quarter of $2 \pi$ unless $M=2$. Thus, the name quadrature mirror filter is a misnomer, when $M>2$. However, this has become more or less standard, and there is no reason to change the name as long as we remember its origin.

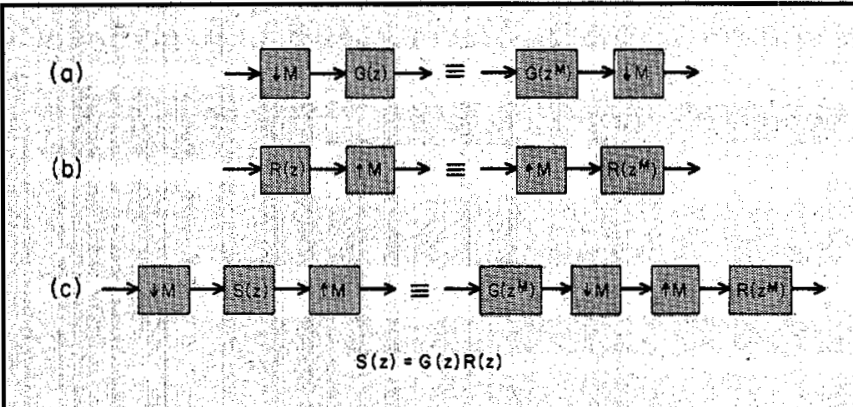

Figure 11. The rules for moving a transfer function around a decimator or interpolator are simple; the figure shows some useful identities. Figure 11 (a) says that a transfer function $G(z)$ following an $M$-fold decimator is equivalent to a transfer function $G\left(z^{M}\right)$ preceding an $M$-fold decimator. Figure $11(\mathrm{~b})$ is the corresponding identity for interpolators. If a transfer function $S(z)$ is sandwiched between a decimator and an interpolator, then the structure can be rearranged as shown in Fig. 11 (c), where $G(z) R(z)$ is any arbitrary factorization of $S[z]$.
If the boldfaced quantities in (17a) are dropped, it is equivalent to dropping the decimators and interpolators. The term corresponding to $n=0$ represents the genuine output which would result if the decimators and interpolators were absent. The terms corresponding to $1 \leq n \leq M-1$ are unwanted aliasing terms. If we wish to cancel aliasing completely, we should choose $F_{k}(z)$ such that these terms are equal to zero for every possible input signal $x(n)$. Assuming that aliasing is somehow canceled, we have the relation

$$
\frac{\hat{X}(z)}{X(z)}=T(z)=\frac{1}{M} \sum_{k=0}^{M-1} H_{k}(z) F_{k}(z)
$$

where $T(z)$ represents the overall transfer function, or the distortion function. If $H_{k}(z)$ and $F_{k}(z)$ are such that $T(z)$ is allpass, then there is no amplitude distortion, on the other hand, if you force $T(z)$ to be a linear phase FIR function, there is no phase distortion. Finally, if $T(z)$ is a pure delay, (and if aliasing has already been canceled) then we have a perfect-reconstruction QMF bank. For the two-channel case, we saw that perfect-reconstruction is a tricky issue, the solution being not really obvious. For the $M$-band case, it is even more challenging. Our purpose here is to indicate a few steps towards the solution to the perfectreconstruction problem for arbitrary $M$. A scheme shall eventually be presented, which is an attractive candidate for study and research.

Equation (17a) gives rise to $M$ equations, which can be looked upon as conditions for perfect reconstruction. These in turn can be written in matrix form:

$$
H(z) f(z)=v
$$

where $f(z)=\left[F_{0}(z) F_{1}(z) \ldots F_{M-1}(z)\right]^{T}$ $V=\left[c z^{-n_{0}} 00 \ldots .00\right]^{T}$, and $\mathbb{H}(z)$ has elements $H_{n, k}=$ $H_{k}\left(z W^{n}\right)$. The matrix $H(z)$ is known as the alias-cancelation matrix (AC-matrix for short). In principle, inversion of this matrix leads to a solution for the synthesis filters $F_{k}(Z)$ in terms of the analysis filters $H_{k}(z)$. However, there are problems associated with such an approach: first, the resulting synthesis filters are unlikely to be stable for a given set of $H_{k}(z)$. It is a tricky issue to try to restrict $H_{k}(z)$ to be such that the above approach gives rise to stable synthesis filters. Second, even if they are stable, they tend to have very high orders. Finally, since $\mathbf{H}(\mathbf{z})$ is a function of $\mathbf{z}$ rather than a constant, its inversion is difficult. Accordingly, there is considerable interest in finding other procedures to attack the perfect reconstruction problem.

\section{A Couple of Useful Identities}

In Fig. 1(a), imagine that we wish to pass the decimated signal $y(n)$ through a delay $z^{-1}$. This is equivalent to delaying the signal $x(n)$ by $M$ units and then decimating the result. Extending this idea further, we can show that a transfer function $G(z)$ following a decimator (Fig. 11(a)) can be equivalently moved to the left of the decimator, as long as each $z$ is replaced with $z^{M}$. In an analogous manner, a transfer function $R(z)$ preceding an interpolator can be moved to the right by replacing $z$ with $z^{M}$, as shown in 
Fig. 11(b). These identities are exact, and can be proved based on the input-output relations of decimators and interpolators.

Now consider Fig. 11(c), where $S(z)$ is a transfer function sandwiched between a decimator and an interpolator. Let $S(z)=C(z) R(z)$ be an arbitrary factorization. Then we can move $G(z)$ and $R(z)$ around to obtain the equivalent diagram shown in the figure. Such manipulations are very useful in obtaining a quick understanding of certain important issues in the QMF problem.

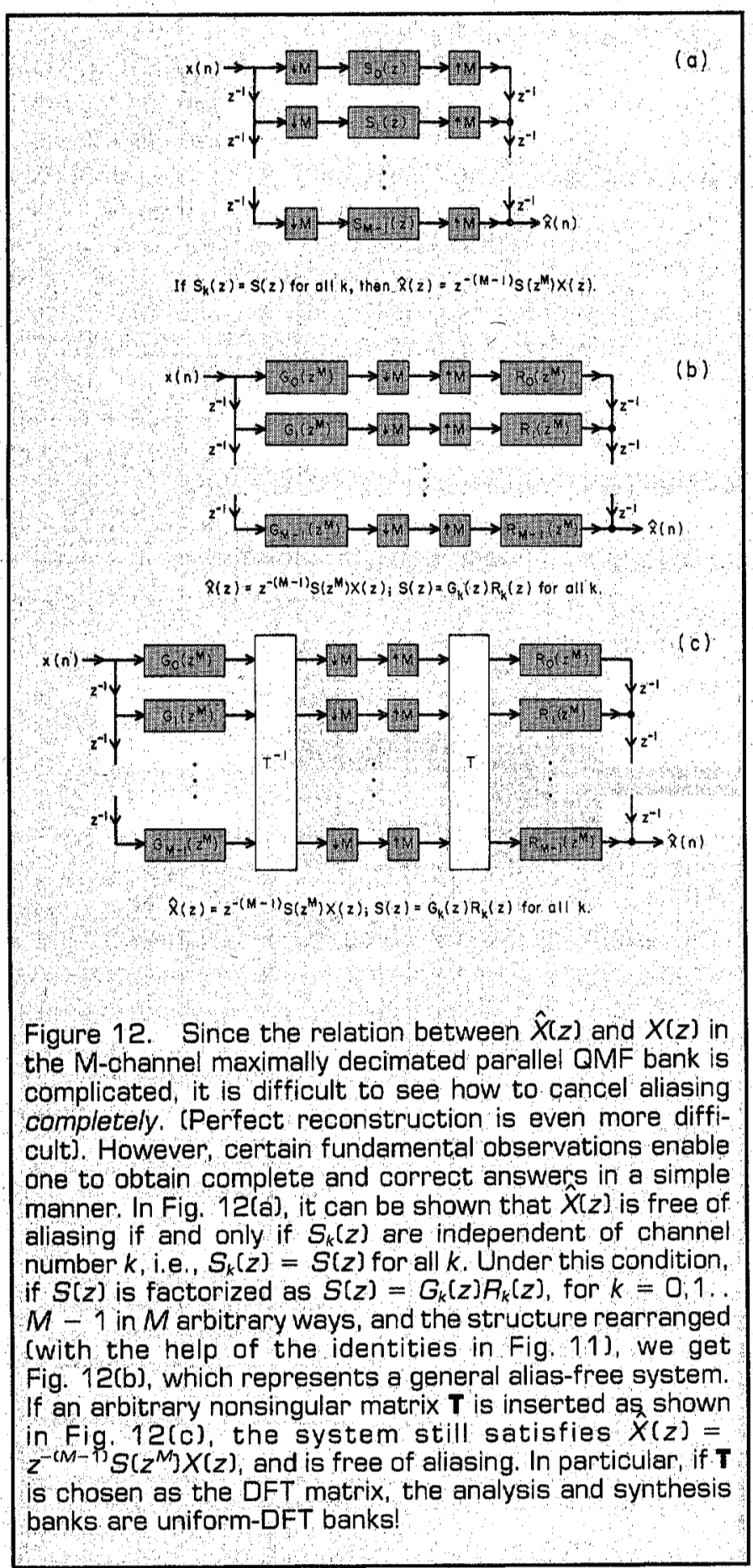

\section{A General M-Band Alias-Free System}

For a minute, let us switch our minds to a completely different picture, viz. Fig. 12. Figure 12(a) shows a set of transfer functions $S_{0}(z), S_{1}(z), \ldots S_{M-1}(z)$ sandwiched between $M$-fold decimators and interpolators. The decimators are preceded by a chain of delays; whereas the interpolators are followed by a chain of delays. Since decimation is not preceded by filtering, there is in general severe aliasing in the structure, for arbitrary $x(n)$. However, it may be possible to pick the functions $S_{k}(z)$ such that the aliasing is somehow canceled by the imaging effect of the interpolators. Let us probe deeper into this possibility.

First, suppose that $S_{k}(z)$ are not there (i.e., $S_{k}(z)=1$ for all $k$.) Then the decimators and interpolators do not do any harm to the signal $x(n)$; we can formally prove in this case [37], that $\hat{x}(n)=x(n-M+1)$. Next suppose that $S_{k}(z)$ are present, but the following relation holds:

$$
S_{k}(z)=S(z), \quad \text { for all } k
$$

i.e., $S_{k}(z)$ is independent of $k$, but otherwise arbitrary. By making use of the identity (b) in Fig. 11, we can move $S(z)$ past the interpolators, and then past the delay chain, to show that $\hat{X}(z)=z^{-(M-1)} S\left(z^{M}\right) X(z)$. Thus; the system is represented by a transfer function $T(z)=z^{-(M-1)} S\left(z^{M}\right)$, and is therefore (linear and) time-invariant. In particular, aliasing has been completely canceled. More formally, it is possible to prove that, in Fig. $12(a), \hat{x}(n)$ is free from aliasing if and only if (19) holds.

Let us now assume that (19) holds, and factorize $S(z)$ in $M$ arbitrary ways:

$$
S(z)=G_{k}(z) R_{k}(z)
$$

By making use of the identities in Fig. 11, we can then redraw Fig. 12(a) as in Fig. 12(b). If we now insert an arbitrary nonsingular constant matrix $T$ and its inverse into the structure as shown in Fig. 12(c), an observer observing $\hat{x}(n)$ will not even know that we have inserted these! In other words, for a given $x(n), \hat{x}(n)$ in Fig. 12(a) is exactly same as $\hat{x}(n)$ in Fig. 12(c), no matter what $T$ is. Accordingly, as long as $G_{k}(z) R_{k}(z)$ is independent of $k, \hat{x}(n)$ in Fig. 12(c) is entirely free of aliasing for any $T$. The effective analysis and synthesis filters in Fig. 12(c) can be verified to be

$$
\begin{aligned}
& H_{k}(z)=\sum_{l=0}^{M-1} z^{-}\left[\mathrm{T}^{-1}\right]_{k l} G_{l}\left(z^{M}\right), \\
& F_{k}(z)=\sum_{l=0}^{M-1} z^{-(M-1-1)} \mathbf{T}_{l k} R_{l}\left(z^{M}\right)
\end{aligned}
$$

Based on this observation, how do we construct some useful QMF banks? As a possible example, let us imagine that $\mathbf{T}$ is related to the DFT matrix, i.e., $\mathbf{T}_{m n}=W^{m n} / M$ where $W=e^{-2 \pi j / M}$. In particular, this means,

$$
\begin{aligned}
H_{0}(z)= & {\left[G_{0}\left(z^{M}\right)+z^{-1} G_{1}\left(z^{M}\right)\right.} \\
& \left.+z^{-2} G_{2}\left(z^{M}\right)+\ldots+z^{-(M-1)} G_{M-1}\left(z^{M}\right)\right]
\end{aligned}
$$

and $H_{k}(z)=H_{0}\left(z W^{k}\right)$. In other words, we have the following situation: suppose somebody gives us a lowpass 


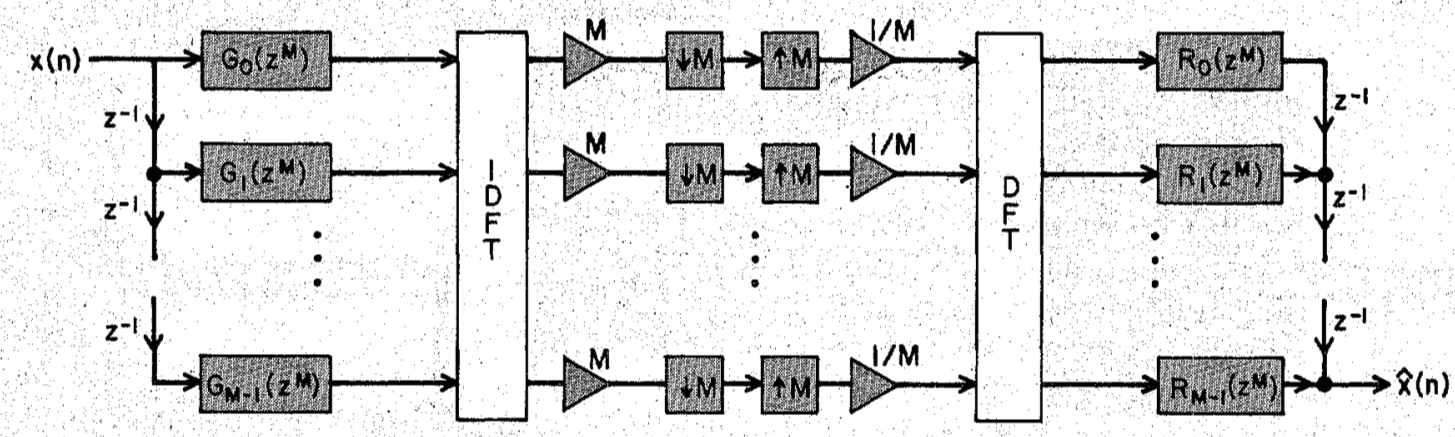

Figure 13, In this figure, the analysis and synthesis banks are of the uniform DFT type. The lowpass filter $H_{0}(z)$ is given by $G_{0}\left(z^{M}\right)+z^{-1} G_{1}\left(z^{M}\right)+$, . $+z^{-(M-1)} G_{M-1}\left(z^{M}\right)$ whereas $H_{k}(z)=H_{0}\left(z W^{k}\right)$. With $R_{k}(z)$ defined as in (25), aliasing is completely canceled, and the system is characterized by the transfer function $T(z)=z^{-M-1\}} \Pi_{f=0}^{M-1} G_{l}\left(z^{M}\right)$. Such schemes for alias cancelation, though perfect, give rise to complicated synthesis filters (in terms of filter order], In practice, if aliasing due to nonadjacent channels is negligible, certain elegant and practical solutions are possible as shown by Rothweiler [9] and Chu [8]. Note that, both in this figure and in Fig. 12, if $R_{k}(z)$ are chosen to be equal to $1 / G_{k}(z)$, we would have perfect reconstruction, and $\hat{x}(n)=$ $x[n-M+1]$. However, unless all the zeros of the numerator polynomials of $G_{k}(z)$ are strictly inside the unit circle (i.e., these numerators are minimum-phase polynomials] $R_{k}(z)$ become unstable, So, such perfect reconstruction structures are not very useful. transfer function

$$
H_{0}(z)=\sum_{n=0}^{\infty} h(n) z^{-n}
$$

We can write $H_{0}(z)$ in the form (22) simply by defining ${ }^{*}$

$$
G_{l}(z)=h(I)+h(I+M) z^{-1}+h(I+2 M) z^{-2}+\ldots
$$

That is, the impulse responses of $G_{f}(z)$ are decimated versions of the impulse response $h(n)$. Having done so, let us take the remaining analysis filters as $H_{k}(z)=H_{0}\left(z W^{k}\right)$. The frequency responses of $H_{k}(z)$ are uniformly shifted versions of the prototype $H_{0}\left(e^{j \omega}\right)$, i.e., $H_{k}\left(e^{j \omega}\right)=$ $H_{0}\left(e^{j(\omega-(2 \pi k / M))}\right)$. Such a set of analysis filters is very commonly used; the analysis bank is then called the uniform DFT bank. Now, what we learned from the exercise of Fig. 12 is that, in a QMF bank with such analysis filters, we can completely eliminate aliasing simply by choosing the synthesis filters $F_{k}(z)$ to be as in (21) with $T_{j k}=W^{i k} / M$, and with $R_{k}(z)$ such that the product $G_{k}(z) R_{k}(z)$ is independent of $k$. For example we could choose

$$
R_{k}(z)=\prod_{\substack{l=0 \\ l \neq k}}^{M-1} G_{l}(z)
$$

or, as an alternative,

$$
R_{k}(z)=1 / G_{k}(z)
$$

With the choice (25) it is clear that the synthesis filters are stable as long as the analysis filters are stable, but the disadvantage is that the transfer functions $F_{k}(z)$ tend to have much higher orders than $H_{k}(z)$. The choice in (26)

\footnotetext{
${ }^{\ddagger} G_{i}(z)$ are called polyphase components [3] of $H_{0}(z)$.
}

overcomes this problem and in addition leads to perfect reconstruction since $S(z)=1$ here; but it does not give rise to stable synthesis filters, unless the numerators of the polyphase components $C_{k}(z)$ have minimum phase.

\section{PERFECT RECONSTRUCTION M-CHANNEL QMF BANKS}

For an $M$-band QMF bank with arbitrary $M$, we saw at least one technique for obtaining perfect reconstruction, namely Fig. 12(c) or Fig. 13 with $R_{k}(z)$ as in (26). As pointed out, such a scheme works under the constraint that $G_{k}(z)$ should have minimum-phase numerators. A different scheme is now outlined, which is free from such a requirement. Consider again the structure of Fig. 12(a), redrawn in Fig. $14(\mathrm{a})$, with $S_{k}(z)=1$ for all $k$. This is then an ultrasimple QMF bank, with $H_{k}(z)=z^{-k}$ and $F_{k}(z)=z^{-(M-1-k)}$ We know from earlier discussions that $\hat{x}(n)=x(n-$ $M+1$ ), i.e., the structure has perfect-reconstruction property, Let us now insert the matrices $\mathbf{R}$ and $\mathbf{R}^{+}$into the structure as shown in Fig $14(b)^{\ddagger}$, where $\mathbf{R}$ is a $M \times M$ unitary matrix (i.e, any matrix satisfying $\mathbf{R}^{+} \mathbf{R}=c \mathbf{l}$ ), where $c$ is a scalar. Evidently, this does not affect the output $\hat{x}(n)$ since the matrices $\mathbf{R}$ and $\mathbf{R}^{\boldsymbol{t}}$ simply cancel. Since $\mathbf{R}$ is memoryless, we can obviously move the matrices to obtain the perfect reconstruction system of Fig. 14(c). This works for any unitary $\mathbf{R}$; the only disadvantage is that the FIR filters $H_{k}(z)$ and $F_{k}(z)$ in Fig. 14(c) are of order $M-1$. In order to accomplish higher orders, and hence sharper filters, let us extend this idea further. Thus, refer to Fig. 14(d) which is obtained from Fig. 14(a) by inserting the matrix functions $\mathbf{E}(z)$ and $\mathrm{E}^{\top}\left(z^{-1}\right)$. The matrix $\mathrm{E}(z)$ is unitary

* Superscript + stands for transposed conjugate and superscript $T$ stands for transpose. 
on the unit circle of the $z$-plane, i.e.,

$$
\mathbf{E}^{T}\left(z^{-1}\right) \mathbf{E}(z)=c \mathbf{l}, \quad \text { for } z=\mathrm{e}^{j \omega},
$$

where $c$ is a scalar constant. (Note that, assuming $E(z)$ is real for real $z, \mathbf{E}^{T}\left(z^{-1}\right)$ is precisely the transpose-conjugate of $\mathrm{E}(z)$ on the unit circle). If $\mathrm{E}(z)$ has complex coefficients, then $\mathbf{E}^{\top}\left(z^{-1}\right)$ should be replaced with $E_{*}^{\top}\left(z^{-1}\right)$ where subscript ' $*$ ' means coefficient conjugation. As a result, the output $\hat{x}(n)$ in Fig. 14(d) continues to be same as that in Fig. 14(a), except for a scaling constant. We can now invoke the identities in Fig. 11, and rearrange Fig. 14(d) as in Fig. 14(e), which is therefore a perfect reconstruction system! As such, unless $E(z)$ is $F I R, E^{T}\left(z^{-1}\right)$ is unstable, so we assume $E(z)$ is FIR. To avoid non causal operations, in practice, we insert a delay in front of $E^{\top}\left(z^{-1}\right)$ so that there are no positive powers of $z$ anywhere. From Fig. 14(e) you can deduce that the analysis and synthesis filters are effectively

$$
H_{k}(z)=\sum_{i=0}^{M-1} z^{-1} E_{k l}\left(z^{M}\right)
$$

and

$$
F_{k}(z)=z^{-\beta} H_{k}\left(z^{-1}\right)
$$

where $\beta$ is a large enough positive integer to ensure that there are no positive powers of $z$ in $F_{k}(z)$.

Now, if (27) holds everywhere on the unit circle, then it must be true for all $z$, by analytic continuation. Such matrices $\mathbf{E}(z)$ are said to be paraunitary ${ }^{\ddagger}$. For our discussion, 'paraunitary' will therefore be used as a synonym to 'unitary on the unit circle'. We can thus state the following result: let $H_{k}(z)$ be FIR analysis filters with polyphase components $E_{k l}(z)$ such that the matrix $E(z)=\left[E_{k l}(z)\right]$ is para-

* The concept of paraunitariness is well-known in classical, continuous-time network theory; scattering matrices that describe lossless multiports satisfy this property [19], [20].

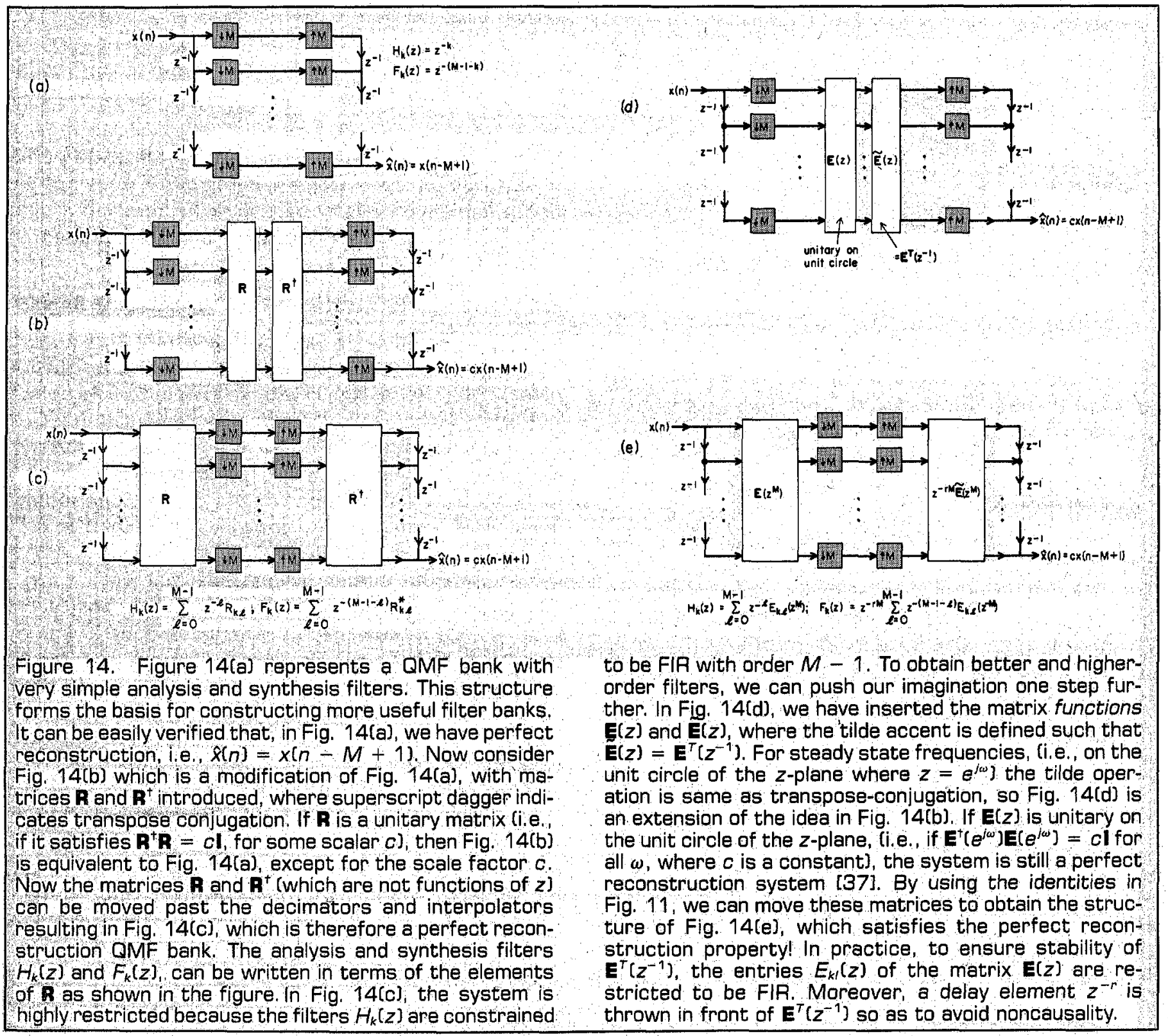


unitary. If we pick the synthesis filters to be as in (28b) then aliasing is completely canceled, and there are no amplitude and phase distortions, i.e., the structure has perfect reconstruction property.

So! Perfect-reconstruction for QMF banks, in principle, is as simple as that! Now comes the important question: how do we construct an $M \times M$ matrix of FIR functions such that it is paraunitary? One procedure would be to construct $E(z)$ as a product of simple unitary building blocks, as indicated in Fig. 15(a), In this figure, $\mathbf{K}_{n}$ are constant $M \times M$ orthogonal matrices (a unitary matrix with real entries is called an orthogonal matrix). The matrices $\Lambda_{n}(z)$ are diagonal matrices of delays, so that they are unitary on the unit circle. Since a product of unitary matrices is unitary, the cascade in Fig. 15(a) realizes the desired $\mathbf{E}(z)$. More general methods for synthesizing $\mathbf{E}(z)$ can be found in [41].

\section{The Design Problem}

What are the design issues now? In practice, we wish $H_{k}(z)$ to have good stopband attenuation, so that adjacent frequency bands are well isolated (this is important, even though aliasing is eventually canceled anyway, because,

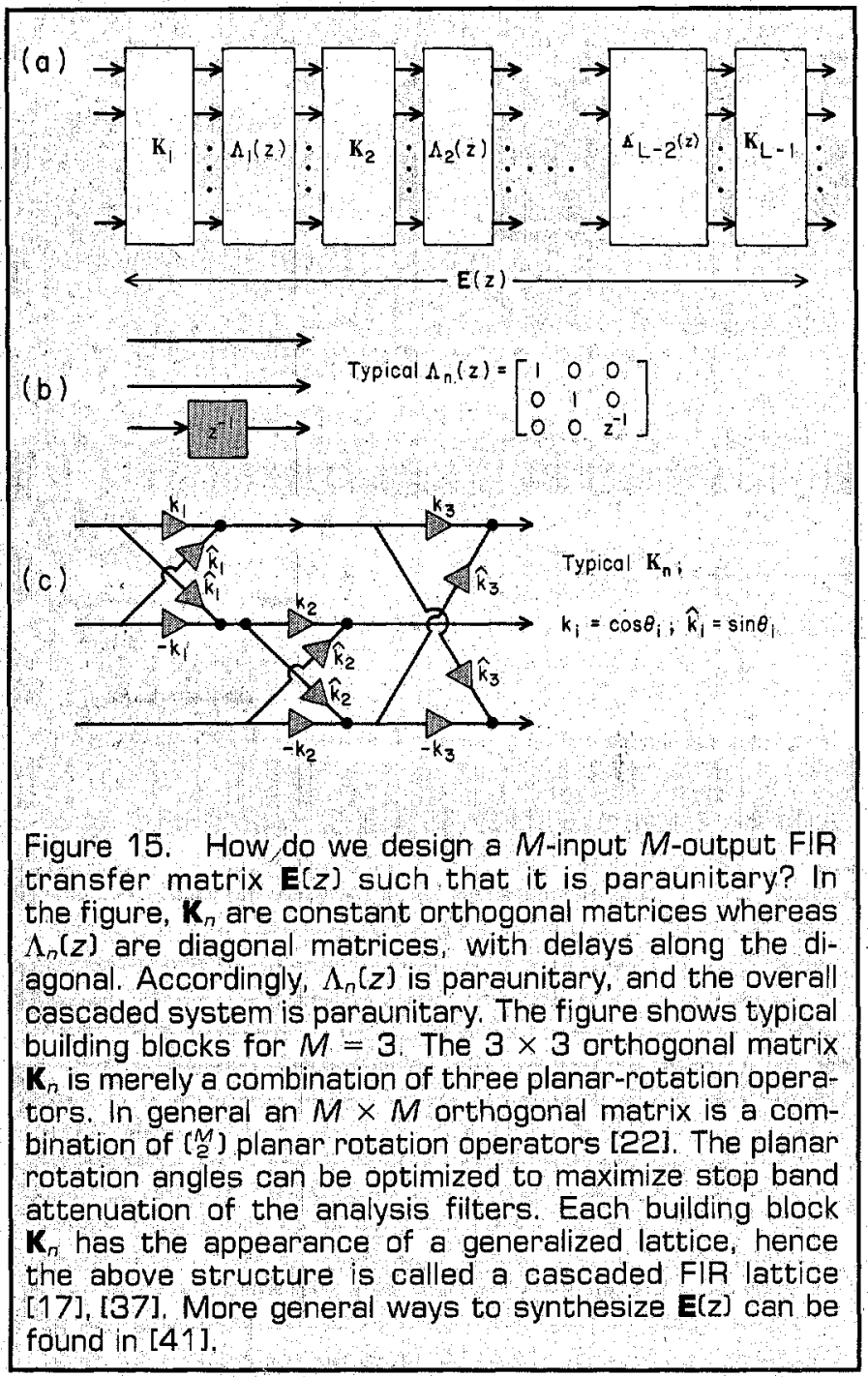

when the subband signals are encoded, maximum freedom from adjacent channels is desired [3]). So the filters $H_{k}(z)$ should have good stopband attenuation. We can now set up an optimization problem: find the orthogonal matrices in Fig. 15(a) such that the stopbands of the analysis filters have minimum possible energy. We need not worry about the passbands because the unitary nature of $\mathbf{E}(z)$ ensures that $\left|H_{0}\left(\mathrm{e}^{j \omega}\right)\right|^{2}+\mid H_{1}\left(\mathrm{e}^{j \omega}\right)^{2}+\ldots+$ $\left|H_{M-1}\left(\mathrm{e}^{j \omega}\right)\right|^{2}=$ constant for all $\omega$. Accordingly, if the stopbands are good, and if the passbands are defined to be disjoint, then the passbands are automatically good. Figures 15 and 16 provide further discussions and examples on this issue.

An $M \times M$ orthogonal matrix can be represented in terms of $\left(\begin{array}{c}M \\ 2\end{array}\right)$ planar rotations. In Fig. 15, an example of the choice of building blocks is shown for the case of $M=3$. A typical $3 \times 3$ orthogonal matrix, constructed as shown in Fig. 15(c), has $\left(\begin{array}{l}3 \\ 2\end{array}\right)=3$ degrees of freedom which can be adjusted so as to obtain good stopband attenuation. The criss-cross nature of the orthogonal building block suggests the name lattice structures for these circuits. A meaningful objective function to be minimized would be the sum of the stopband energies of the analysis filters. Fig. 16 shows a design example for the case of $M=3$, with $L-1=31$. Figure $16(b)$ shows the optimized frequency response plots. The transfer functions $H_{0}(z), H_{1}(z)$ and

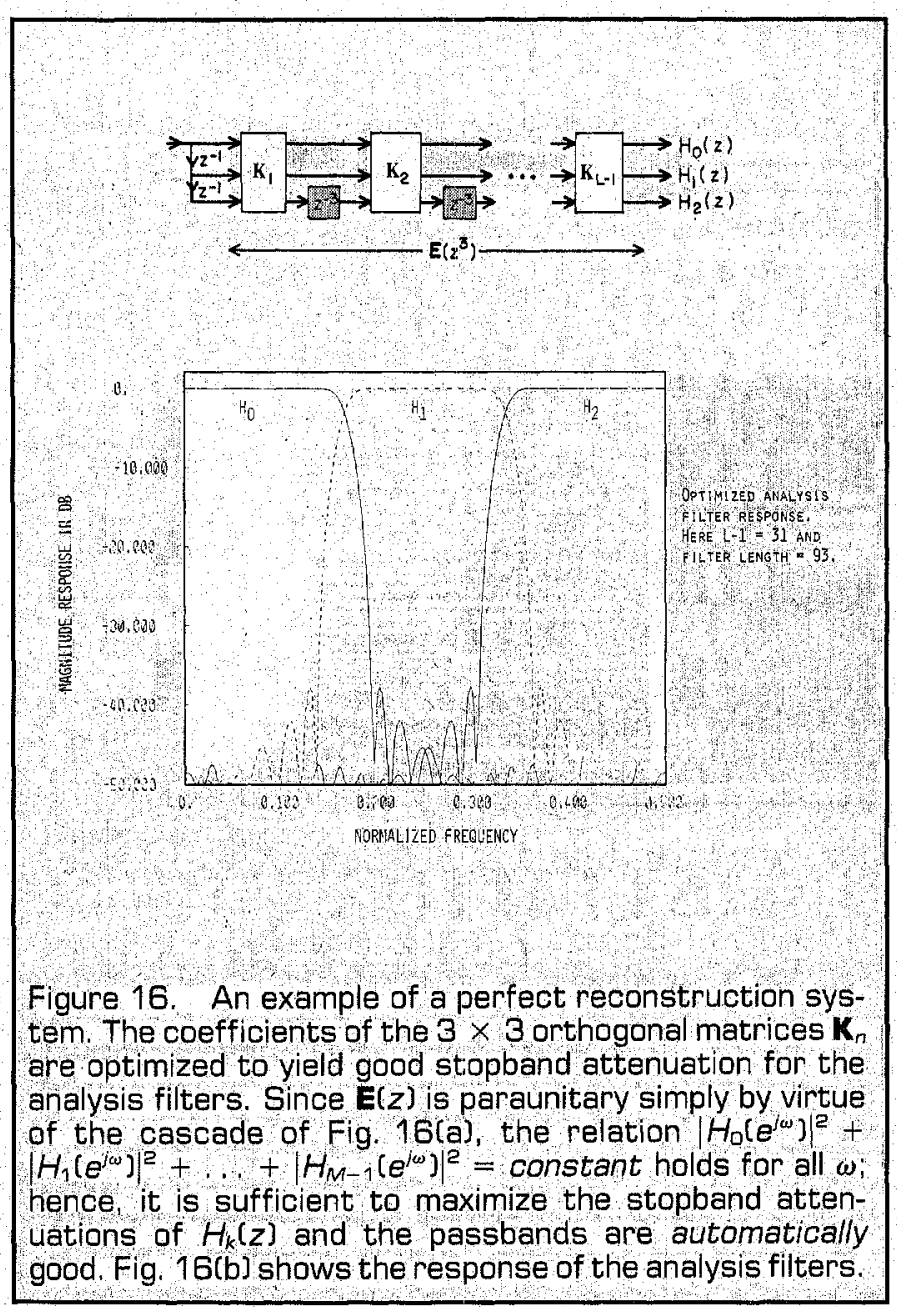


$H_{2}(z)$ have orders $N-1=3(L-2)+2=92$. The optimization of the lattice coefficients was performed using software available in $[23]^{*}$.

In the case of $M=2$, the lattice structure can be drawn in a particularly simple form, by considering a denormalized orthogonal matrix. This is indicated in Fig. 17. Notice that this lattice is different from the linearprediction lattice structure because of the minus sign on one of the $\alpha^{\prime} s$. This sign-difference is very crucial, and is what enables us to employ the structure for the QMF application.

This lattice structure has several unique properties [42]. First, the transfer functions $H_{0}(z)$ and $H_{1}(z)$ generated by the lattice satisfy the condition of equation (16); second, the synthesis-bank lattice, which is the transpose of the analysis bank has transfer functions $F_{0}(z)$ and $F_{1}(z)$ satis. fying (16). Moreover, $H_{0}(z)$ is a spectral factor of a linearphase halfband FIR filter. Finally, the condition of equation (15) is satisfied. These four properties hold, regardless of the values of $\alpha_{k}$ used in the structure! (The quantities $\alpha_{k}$ determine only the sharpness of cutoff and stopband attenuation of $\left.H_{0}(z)\right)$. In summary, if these lattice structures are used in the analysis and synthesis banks of the two-channel QMF circuit, then perfect reconstruction is guaranteed even if $\alpha_{k}$ are quantized to arbitrarily small number of bits (in a digital implementation). In other words, perfect-reconstruction is structurally induced by the lattice.

F This example was generated by Truong Q. Nguyen, at Caltech.

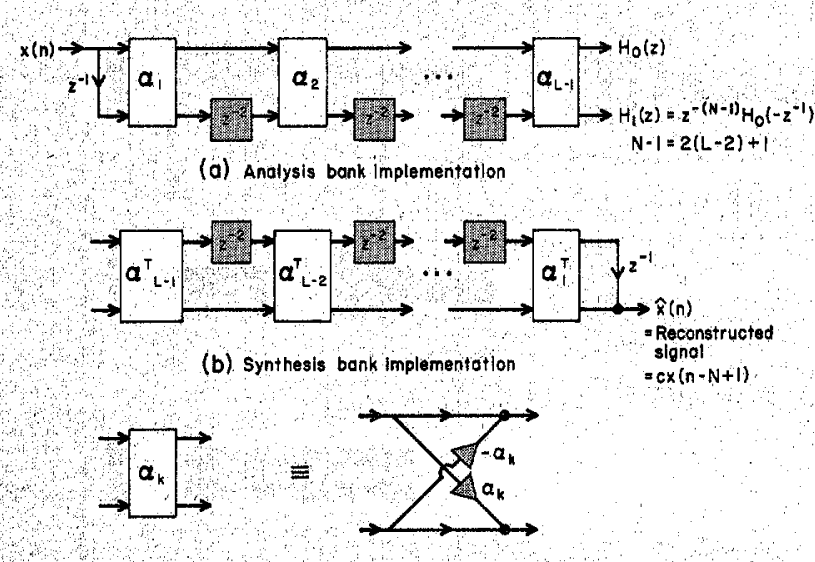

(c) The lattice-structure bullding block

Figure 17. In the two-channel lattice structure, regardless of the values of $\alpha_{k}$, the following property holds: $\hat{x}(n)=c x(n-N+1$ ) (perfect reconstruction). The lattice structure is therefore robust [42] and is attractive for implementation purposes. In particular, we have perfect reconstruction even if $\alpha_{k}$ are quantized to mere powers of two! lif the transfer functions $H_{k}(z)$ and $F_{k}(z)$ were implemented in direct-form, then coefficient quantization would have destroyed the perfectreconstruction property]. Notice that the above lattice [17] is different from the linear-prediction lattice [36], because of the minus sign in the criss-cross.
This unique nature of the lattice encourages us to design the two-channel perfect reconstruction analysis filter $H_{0}(z)$ in a different way rather than by spectralfactorizing a half-band filter (see Fig. 18). We simply formulate an objective function

$$
P=\int_{\omega_{S}}^{\pi} \mid H_{0}\left(e^{j \omega) / 2 d \omega}\right.
$$

and find the set of parameters $\alpha_{k}$ that minimizes $P$. The lattice structure automatically ensures the rest; in particular the passband of $H_{0}(z)$ comes out to be good because, firstly, $\left|H_{1}\left(e^{j \omega}\right)\right|$ is an image of $\left|H_{0}\left(e^{j \omega}\right)\right| \cdot$ (because of (16)), and furthermore, $\left|H_{0}\left(\mathrm{e}^{j \omega}\right)\right|^{2}+\left|H_{1}\left(\mathrm{e}^{j \omega}\right)\right|^{2}=$ constant holds. In Fig. 19, we can see plots of $\left|H_{0}\left(\mathrm{e}^{j \omega}\right)\right|$ and $\left|H_{1}\left(\mathrm{e}^{j \omega}\right)\right|$ obtained by such an optimization ${ }^{\ddagger}$. The reconstruction error is expected to be zero. Figure 19 (b) shows a plot of $\left|T\left(e^{j \omega}\right)\right|$ with the lattice coefficients quantized to 5 bits. Because of structural perfect-reconstruction property, the plot is constant for all $\omega$. Instead of quantizing the lattice coefficients, if we quantize the direct-form coefficients, the resulting $\left|T\left(e^{j \omega}\right)\right|$ is not completely flat, because the quantized $H_{0}(z)$ is then no longer a spectral factor of a half-band filter. The lattice structure in Fig. 17, therefore, seems to be desirable both from the viewpoint of designing $H_{0}(z)$ and from the viewpoint of implementing the analysis and synthesis banks.

It is worth pointing out yet another feature of the lattice structure, viz., the modular property. What we mean by this is the following: suppose we drop (i.e., simply eliminate) the lattice sections labeled $\alpha_{L-1}$ and $\alpha_{L-1}^{T}$ along with

\footnotetext{
F This design example was generated by Phuong-Quan Hoang at
} Caltech.

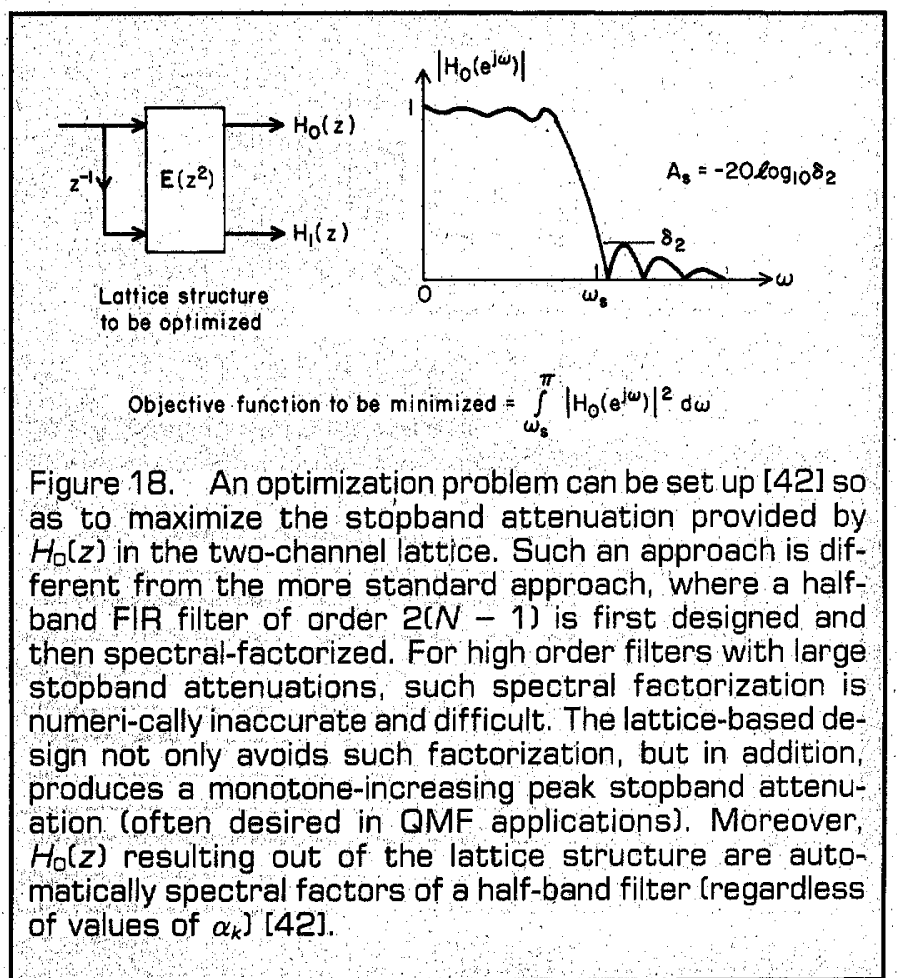


the associated delays $\left(z^{-2}\right.$ elements). Then the resulting smaller lattice structure continues to have perfect reconstruction property. In other words, the effect of adding more sections or deleting sections is to change the attenuation characteristics of $H_{0}(z)$, but the signal $\hat{x}(n)$ continues to be a perfect replica of $x(n)$ except for a delay. Such a modular property is of course, not available with the direct-form structure.

\section{CONCLUDING REMARKS}

The purpose of this paper has been to outline some of the issues involved in the QMF-bank design problem. The relation of the QMF problem to the concept of losslessness (or unitariness) has been emphasized in this article. It is encouraging to know that perfect-reconstruction can be accomplished for arbitrary number of channels in .

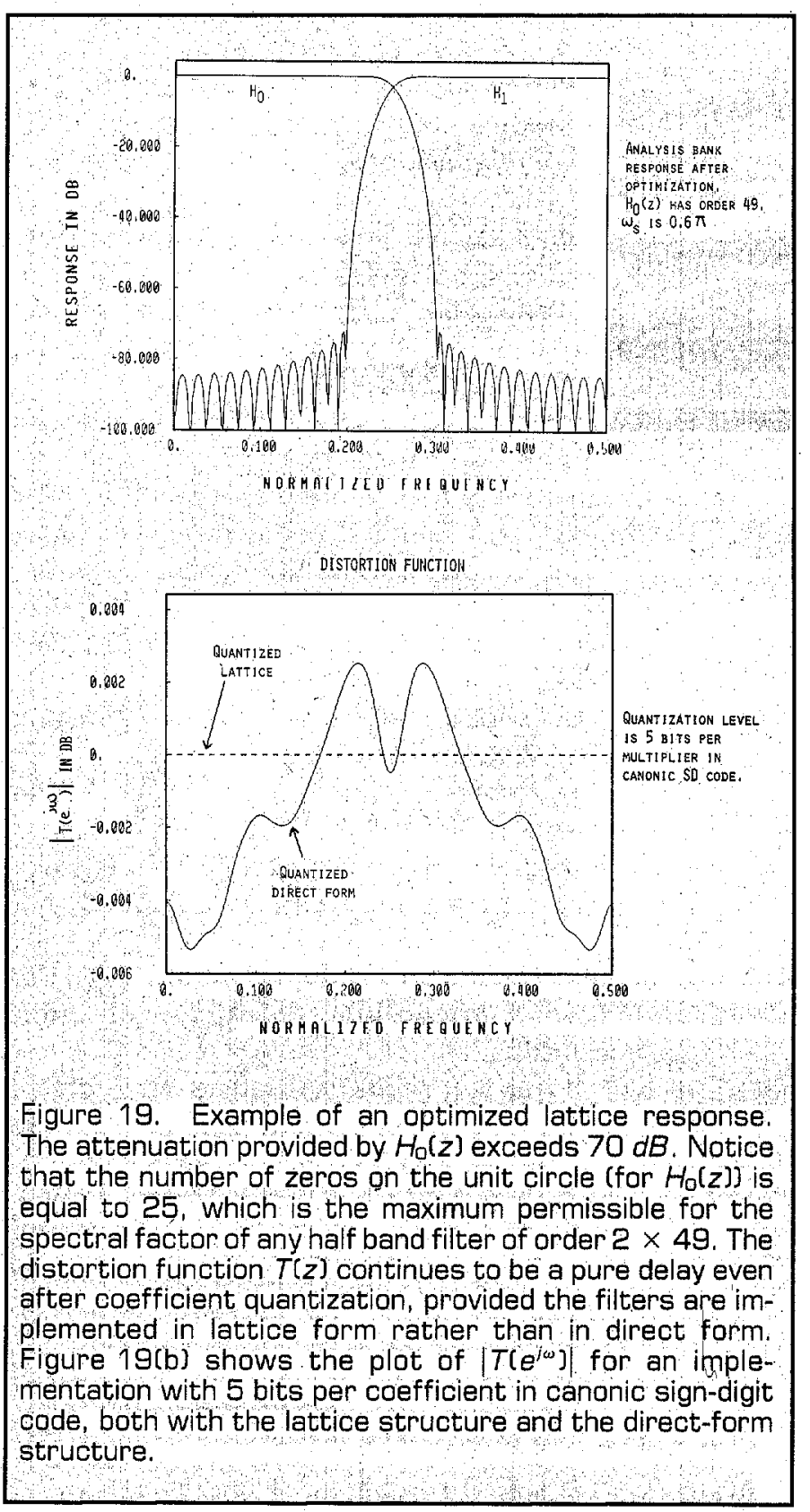

a maximally decimated parallel QMF bank. Moreover, such reconstruction is possible even when the analysis filters $H_{k}(z)$ and synthesis filters $F_{k}(z)$ are all FIR, and of the same length $N$. Certain new lattice structures, fundamentally different from the linear-prediction lattice, are naturally placed in evidence when we attempt to do perfect reconstruction based on unitary building blocks. Moreover, for the case of two channels, these lattice structures give rise to transfer functions which have all the properties required for perfect reconstruction even when the lattice coefficients are quantized to arbitrarily small number of bits.

\section{ACKNOWLEDGMENTS}

I wish to express my thankfulness to Drs. N.S. Jayant and R.E. Crochiere of the AT\&T Bell Laboratories, for encouraging me to write this article. It was Ron Crochiere who triggered the author's interest in multirate DSP three years ago: I take this opportunity to thank Professors Smith and Barnwell of the Georgia Institute of Technology, for useful discussions on the QMF problem. Thanks are also due to Dr. Martin Vetterli of the Ecole Polytechnique Federale de Lausanne, and to Prof. T: Ramstad of the Norwegian Institute of Technology, for fruitful comments on the topic of this paper. I also wish to thank Truong Q. Nguyen, Ms. Zinnur Doganata and Ms. Phuong-Quan Hoang, graduate students at Caltech, for interaction on the one dimensional perfect-reconstruction problem, and for producing impressive design examples. Finally, the enthusiasm and interest shown by Dr. Kumar Swaminathan of the AT\&T Bell Labs (former Caltech student) is gratefully acknowledged.

\section{REFERENCES}

[1] A. Croisier, D. Esteban, and C. Galand, "Perfect channel splitting by use of interpolation/decimation/tree decomposition techniques," International Conf. on Information Science and Systems, Patras, Greece, 1976.

[2] D. Esteban and C. Galand, "Application of quadrature mirror filters to split-band voice coding schemes," Proc. of the IEEE International Conf. ASSP, Hartford, Connecticut, pp. 191-195, May 1977.

[3] R. E. Crochiere and L. R. Rabiner, Multirate Digital Signal Processing, Englewood Cliffs, NJ: PrenticeHall, 1983.

[4] V. K. Jain and R. E. Crochiere, "Quadrature mirror filter design in the time domain," IEEE Trans, on Acoustics, Speech, and Signal Processing, vol. ASSP32, pp. 353-361, April 1984.

[5] C. R. Galand and H.J. Nussbaumer, "New quadrature mirror filter structures," IEEE Trans. on Acoustics, Speech, and Signal Processing, vol. ASSP-32, pp. 522-531, June 1984.

[6] M. J. T. Smith and T. P. Barnwell III, "A procedure for designing exact reconstruction filter banks for tree structured subband coders," IEEE International Conf. on Acoustics, Speech, and Signal Processing, 
pp. 27.1.1-27.1.4, San Diego, California, March 1984.

[7] M.J.T. Smith and T.P. Barnwell III, "A unifying framework for analysis/synthesis systems based on maximally decimated filter banks," Proc. IEEE International Conf. ASSP, Tampa, Florida, pp. 521-524, March 1985.

[8] P. L. Chu, "Quadrature mirror filter design for an arbitrary number of equal bandwidth channels," IEEE Trans. on Acoustics, Speech, and Signal Pro: cessing, vol. ASSP-33, pp. 203-218, February 1985.

[9] J: H. Rothweiler, "Polyphase quadrature filters-A new subband coding technique," Proc. of the 1983 IEEE International Conf. on ASSP, pp. 1280-1283, Boston, Massachusetts, March 1983.

[10a] K. Swaminathan and P. P. Vaidyanathan, "Theory of uniform DFT, parallel quadrature mirror filter banks," IEEE International Conf. on ASSP, Tokyo, Japan, April 1986.

[10b] K. Swaminathan and P. P. Vaidyanathan, "Design of uniform DFT, parallel FIR quadrature mirror filters," IEEE International Symposium on Circuits and Systems, San Jose, California, May 1986.

[11] K. Swaminathan and P. P. Vaidyanathan, "Theory and design of uniform DFT, parallel quadrature mirror filter banks," IEEE Trans: on Circuits and Systems, December 1986 Vol. CAS-33, pp. 1170-1191.

[12a] M. Vetterli, "Splitting a signal into subsampled channels allowing perfect reconstruction," Proc. of the IASTED Conf. on Applied Signal Processing and Digital Filtering, Paris, France; June 1985.

[12b] M. Vetterli, "Filter banks allowing for perfect reconstruction," in Signal Processing, Elsevier Science Publishers, North Holland, pp. 1-26, 1986.

[13] F. Mintzer, "Filters for distortion-free two-band multirate filter banks," IEEE Trans. on Acoustics, Speech, and Signal Processing, vol: ASSP-33, pp. 626-630, June 1985.

[14] P. P. Vaidyanathan and S. K. Mitra, "Low passband sensitivity digital filters: a generalized viewpoint and synthesis procedures," Proc. of the IEEE, pp. 404-423, April 1984.

[15] P. P. Vaidyanathan, "New cascaded lattice structures for FIR filters having extremely low coefficient sensitivity," IEEE International Conf. on Acoustics, Speech, and Signal Processing, pp. 497-500, Tokyo, Japan, April 1986.

[16] P. P. Vaidyanathan, "Implementation of arbitrary FIR transfer functions as passive cascaded lattice structures," IEEE International Symposium on Circuits and Systems, San Jose, California, May 1986.

[17] P. P. Vaidyanathan, "Passive cascaded lattice structures for low sensitivity. FIR filter design, with applications to filter banks,". IEEE Trans. on Circuits and Systems, vol. CAS-33, pp. 1045-1064, November 1986.

[18] F. Mintzer, "On half-band, third-band and Nth-band FIR filters and their design," IEEE Trans. on Acoustics, Speech, and Signal Processing, vol. ASSP-30, pp. 734-738, October 1982.

[19] V. Belevitch, Classical Network Synthesis, HoldenDay, San Francisco, California, 1968.

[20] B.D. O. Anderson and S. Vongpanitlerd, Network Analysis and Synthesis, Prentice-Hall, Inc., Englewood Cliffs, New Jersey, 1973.

[21] T. Kailath, Linear Systems, Prentice-Hall Inc., Englewood Cliffs, New Jersy, 1980.

[22] F. D. Murnaghan, The Unitary and Rotation Groups, Spartan Books, Washington, D. C., 1962.

[23] The IMSL Library: a set of FORTRAN subroutines for mathematics and statistics.

[24] P. P. Vaidyanathan, "On power-complementary FIR filters," IEEE Trans. on Circuits and Systems, vol. CAS-32, pp. 1308-1310, December 1985.

[25] M.G. Bellanger, G. Bonnerot, and M. Coudreuse, "Digital filtering by polyphase network: application to sample-rate alteration and filter banks," IEEE Trans. on Acoustics, Speech, and Signal Processing, vol. ASSP-24, pp. 109-114, April 1976.

[26] A. G. Constantinides and R. A. Valenzuela, "An efficient and modular transmultiplexer design," IEEE Trans. on Communications, vol. 30, pp. 1629-1641, July 1982.

[27] A. Fettweis, J.A. Nossek, and K. Meerkotter, "Reconstruction of signals after filtering and sampling rate reduction," IEEE Trans. on Acoustics, Speech, and Signal Processing, vol. ASSP-33, pp. 893-902, August 1985.

[28] T. H. Ramstad, "Analysis/synthesis filter banks with critical sampling," International Conference on Digital Signal Processing, Florence, September 1984.

[29] T. P. Barnwell III, "Subband coder design incorporating recursive quadrature filters and optimum ADPCM coders," IEEE Trans. on Acoustics, Speech, and Signal Processing, vol. ASSP-30, pp. 751-765, October 1982.

[30] R. V. Cox, D. E. Bock, K. B. Bauer, ]. D. Johnston, and J.H. Snyder, "The analog voice privacy system," IEEE International Conference on Acoustics, Speech, and Signal Processing, pp. 341-344, April 1986.

[31] N.S. Jayant and P. Noll, Digital Coding of Waveforms, Prentice-Hall, Inc, Englewood Cliffs, NJ, 1984.

[32] L. R. Rabiner and B. Gold, Theory and Application of Digital Signal Processing, Prentice-Hall, Inc., Englewood Cliffs, NJ.

[33] L. Gaszi, "Explicit formulas for lattice wave digital filters," IEEE Trans. on Circuits and Systems, vol. 32, pp. 68-88, January 1985.

[34] P. P. Vaidyanathan, S. K. Mitra, and Y. Neuvo, "A new approach to the realization of low-sensitivity IIR digital filters," IEEE Trans. on Acoustics, Speech, and Signal Processing, vol. 34, pp. 350-361, April 1986.

[35] J. H. McClellan, T.W. Parks, and L.R. Rabiner, "FIR linear-phase filter design program," in Programs for Digital Signal Processing, New York, IEEE Press, 1979, pp. 5.1.1-5.1.13.

[36] J. D. Markel and A. H. Gray Jr., Linear Prediction of 
Speech, Springer Verlag, New York, 1976.

[37] P. P. Vaidyanathan, "Theory and design of $M$-channel maximally-decimated quadrature mirror filters with arbitrary $M$, having perfect-reconstruction property," Technical Report, California Institute of Technology, April 1986. (Also see IEEE Transactions on ASSP, vol. 35, pp. 476-492, April, 1987.)

[38] A. H. Gray, "Passive cascaded lattice digital filters," IEEE Trans. on Circuits and Systems, vol. 27, 337-344, May 1980.

[39] T. Saramaki, "On the design of digital filters as a sum of two allpass filters," IEEE Trans. on Circuits and Systems, vol. 32, pp. 1191-1193, November 1985.

[40] R. Ansari and B. Liu, "A class of low-noise computationally efficient recursive digital filters with applications to sampling rate alterations," IEEE Trans. on Acoustics, Speech, and Signal Processing, vol. 33, pp. 90-97, February 1985.

[41] P. P. Vaidyanathan, Zinnur Doganata, and Truong Q. Nguyen, "More results on the perfect reconstruction problem in M-band parallel QMF banks," IEEE International Symp. on Circuits and Systems, Philadelphia, May 1987 (to be published).

[42] P. P. Vaidyanathan and Phuong-Quan Hoang, "The perfect-reconstruction QMF bank: new architectures, solutions, and optimization strategies," IEEE International Conference on Acoustics, Speech and Signal Processing, Dallas, pp. 2169-2172, April 1987 (to be published).

[43] P. P. Vaidyanathan, P. Regalia and S. K. Mitra, "Design of doubly complementary IIR digital filters using a single complex allpass filter, with multirate applications," IEEE Trans. on Circuits and Systems, vol. 34, April 1987 (to be published).

[44] P. P. Vaidyanathan and V. Liu, "An improved sufficient condition for absence of limit cycles in digital filters," IEEE Trans. on Circuits and Systems, vol. CAS-34, pp. 319-322, March 1987.

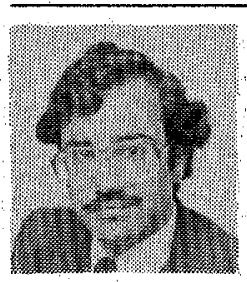

P.P. Vaidyanathan $\left(S^{\prime} 80-M^{\prime} 83\right)$ was born in Calcutta, India, on October 16, 1954, He received the B.Sc. (with honors) degree in physics, and the B. Tech and $M$. Tech. degrees in radiophysics and electronics from the University of Calcutta, India, in 1974, 1977, and 1979, respectively, and the Ph.D. degree in electrical and computer engineering from the University of California, Santa Barbara, in 1982.

He was a Postdoctoral Fellow at the University of California, Santa Barbara, from September 1982 to February 1983. Since March 1983, he has been with the California Institute of Technology, Pasadena, as an Assistant Professor of Electrical Engineering. His main research interests are in digital signal processing, linear systems, and filter design.

Dr. Vaidyanathan served as the Vice-Chairman of the Technical Program Commiftee for the 1983 . IEEE International Symposium on Circuits and Systems. He currently serves as an Associate Editor for the IEEE TRANSACTIONS ON CIRCUITS AND SYSTEMS. He was the recipient of the Award for Excellence in Teaching at the California Institute of Technology for 1983/84. He was also a recipient of the NSF's Presidential Young Investigator Award, starting from the year 1986.

\section{SUMMARY OF KEY RESULTS}

The M-channel Quadrature Mirror Filter (QMF) bank in Fig. 10 is called the maximally decimated, parallel QMF bank. In order to avoid spectral gaps while splitting the signal $x(n)$ into $M$ bands, the frequency responses of the analysis filters $H_{k}(z)$ are permitted to overlap. Consequently, there is aliasing at the output of the decimators. This aliasing can be canceled by the imaging effects of the interpolators, if the synthesis filters $F_{k}(z)$ are chosen appropriately. Some schemes [10], [11] for perfect cancelation of aliasing are shown in Fig. 12 and Fig. 13, and typically require high orders for $F_{k}(z)$. Approximate cancelation of aliasing can be achieved by use of suitable synthesis filters of low order [8], [9].

Once aliasing has been canceled, the reconstructed signal is given by $\hat{X}(z)=T(z) X(z)$ where $T(z)$ is the overall transfer function or the distortion transfer function. If $\left|T \cdot\left(e^{j \omega}\right)\right|$ is constant independent of $\omega$ (i.e., if $T(z)$ is an allpass function) there is no amplitude distortion; if $\arg \left[T\left(e^{j \omega}\right)\right]=K \omega$ (i.e., if $T(z)$ is a linear-phase (FIR) function) then there is no phase distortion. In fact it has been possible in the past to thus eliminate either amplitude distortion or phase distortion completely [1], [5], [10], [11], [29]. Simultaneous elimination of all three distortions (i.e., aliasing, amplitude and phase distortions) is difficult but can be done. Such a QMF structure will be a perfectreconstruction structure and satisfies $\hat{x}(n)=c x\left(n-n_{0}\right)$. If $E_{k, n}(z), 0 \leq n \leq M-1$ represent the $M$ polyphase components of the analysis filters $H_{k}(z), 0 \leq k \leq M-1$, (see discussions around equations (23), (24) for meaning of polyphase components) and if the matrix function

$$
\mathbf{E}(z)=\left[E_{k, n}(z)\right]
$$

is unitary on the unit circle of the $z$-plane (Fig. 14), then it is possible to obtain perfect reconstruction simply by taking the synthesis filters to be

$$
F_{k}(z)=z^{-\beta} H_{k}\left(z^{-1}\right)
$$

where $\beta$ is an integer large enough so that there are no positive powers of $z$ in the expressions for $F_{k}(z)$ 's.

Such perfect reconstruction is practicable provided $H_{k}(z)$ are FIR; notice that, the poles of $H_{k}\left(z^{-1}\right)$ are the reciprocals of those of $H_{k}(z)$, hence if $H_{k}(z)$ are (stable and) IIR, $F_{k}(z)$ given by Eqn. (B) are necessarily unstable. When $H_{k}(z)$ are FIR, the above perfect-reconstruction scheme has FIR synthesis filters $F_{k}(z)$ having the same order as the analysis filters, and there is an ultrasimple closed form expression (Eqn. (B)) that gives $F_{k}(z)$ in terms of $H_{k}(z)$ ! No inversion of matrices and matrix-polynomials are involved in the design. An implementation of such a system is shown in Fig. 14(e), whereas Fig. 14(d) is a more efficient implementation with the polyphase filters $E_{k, n}(z)$ operating at the lowest possible rate.

A matrix which is unitary on the unit circle satisfies $\mathbf{E}^{\top}\left(z^{-1}\right) \mathbf{E}(z)=c$ l for all $z=e^{j \omega}$, where $c$ is a constant scalar. By analytic continuation, this implies $\mathbf{E}^{T}\left(z^{-1}\right) \mathbf{E}(z)=\mathrm{Cl}$ for all $z$. Such matrices will be called paraunitary or simply lossless. The term 'lossless' comes from the fact that if 
$Y(z)=E(z) X(z)$, then the energy in the vector sequence $y(n)$ is equal to that in the vector sequence $x(n)$. In the continuous-time world, such paraunitary systems are wellknown; scattering matrices of lossless multiports are known to have this property [19], [20].

$A$ second way to look at the perfect-reconstruction scheme is through the Alias Cancelation (AC-) matrix $\mathrm{H}(\mathrm{z})$ in Eqn. (18). If this matrix is unitary, then we can solve for the synthesis filter vector $f(z)$ simply by taking $f(z)=z^{-p} H^{T}\left(z^{-1}\right) v$ (where $p$ is large enough so that there are no positive powers of $z$ in the expressions for $\left.F_{k}(z)\right)$.
It turns out that, with this viewpoint, the same solutions viz. Eqn. (B) results and the (para)unitariness of $E(z)$ is equivalent to (para)unitariness of $\mathbf{H}(z)$.

This work was supported in part by the National Science Foundation grants ECS 84-04245 and DCl 8552579, and in part by Caltech's Programs in Advanced Technology grant, sponsored by Aerojet General, General Motors, GTE and TRW.

\title{
ACOUSTICS, SPEECH AND SIGNAL PROCESSING SOCIETY
}

\author{
Call for Adcom Nominations
}

The nominating committee is currently preparing a slate of nominees for next year's Administrative Committee and would welcome suggestions from the society membership. If you would like to serve as a member of AdCom or know someone who you would like to have as a representative, please send this information to the nominating committee chairperson.

All suggestions will be carefully considered; however, a nominating petition with a minimum of 25 names of society membership will guarantee placement of the nominee on the slate of AdCom candidates to be presented to the membership for mail ballot. The deadline for the return of suggestions and petitions is August 14, 1987 .

\section{Nominating Committee Members}

Benjamin Friedlander

Committee Chairman

Saxpy Computer Corporation

255 San Geronimo Way

Sunnyvale, CA 94086

(408) $732-6700$
Monson Hayes

School of Electrical Engineering

Ceorgia Institute of Technology

Atlanta, GA 30332

(404) $894-2958$

James Cádzow

Electrical \& Computer Engineering

Arizona State University

Tempe, AZ 85287

(602) $965-6623$

Gwyn P. Edwards

Racal-Vadic

1525 McCarthy Blvd.

Milpitas, CA 95035

(408) $946-2227 \times 2725$

Dan Dudgeon

MIT - Lincoln Laboratory

P.O. Box 73

Lexington, $\mathrm{MA} 02173$
George W. Johnson

IBM

Federal Systems Division 9500 Godwin Drive

Manassas, VA 22110

(703) $367-4708$

Stephen E. Levinson

Room 2D-528

AT\&T Bell Laboratories 600 Mountain Avenue Murray Hill, NJ 07974 (201) 582-3503

James Snyder

(617) $863-5500 \times 7216$
AT\&T Bell Laboratories 600 Mountain Avenue Murray Hill, NJ 07974

(201) 582-6403 